\title{
A systematic review comparing the results of early vs delayed ligament surgeries in single anterior cruciate ligament and multiligament knee injuries
}

Seong Hwan Kim ${ }^{1,2+}$, Sang-Jin Han ${ }^{1 \dagger}$, Yong-Beom Park ${ }^{2}$, Dong-Hyun Kim², Han-Jun Lee ${ }^{1}$ and Nicolas Pujol ${ }^{3^{*}}$ (i)

\begin{abstract}
Purpose: The purpose of this study was to compare clinical outcomes and incidence of concomitant injuries in patients undergoing early vs delayed surgical treatment of single anterior cruciate ligament $(A C L)$ injury and multiligament knee injury (MLKI).

Methods: A literature search using PubMed, Embase, the Cochrane Library, the Cumulative Index to Nursing and Allied Health, and Scopus from their inception to April 30, 2020 was conducted. Studies with levels I to IV evidence reporting the incidence of meniscus or cartilage injury according to early vs delayed surgery in single ACL injuries and MLKIs were included. In the meta-analysis, data based on the number of meniscus and cartilage injuries were extracted and pooled. Lysholm and Tegner scores were analyzed using two-sample Z-tests to calculate the nonweighted mean difference (NMD). A meta-regression analysis was also performed to determine the effect of single $A C L$ injury and MLKI/study design.
\end{abstract}

Results: Sixteen studies on single ACL injury and 14 studies on MLKI were included in this analysis. In the analysis, there were significant decreases in Lysholm score (NMD - 5.3 [95\% confidence interval (Cl) - 7.37 to - 3.23]) and Tegner score (NMD $-0.25[95 \% \mathrm{Cl}-0.45$ to -0.05$]$ ) and increases in risk of meniscus tear (odds ratio [OR] 1.73 [95\% Cl 1.1-2.73], $p=0.01$ ) and cartilage injury (OR 2.48 [95\% Cl 1.46-4.2], $p=0.0007$ ) in the delayed surgery group regardless of single $A C L$ injury or MLKI. The result of the meta-regression analysis indicated that single ACL injury and MLKI/study design were not significant moderators of overall heterogeneity $(p>0.05)$.

Conclusions: Our study suggests that delayed ACL surgery significantly resulted in a higher risk of meniscus tear and cartilage injury and decreased Lysholm and Tegner scores compared to early ACL surgery. The Lysholm scores in the delayed MLKI surgery group were significantly decreased, but the risks of meniscus tear and cartilage injury in the delayed MLKI surgery group remained unclear.

Level of evidence: Level III, meta-analysis.

Keywords: Early surgery, Delayed surgery, Multiligament knee injury, Anterior cruciate ligament injury, Meta-analysis

\footnotetext{
*Correspondence: npujol@ch-versailles.fr

${ }^{\dagger}$ Seong Hwan Kim and Sang-Jin Han contributed equally to this work.

${ }^{3}$ Department of Orthopedic Surgery, Centre Hospitalier de Versailles, Le Chesnay, France

Full list of author information is available at the end of the article
}

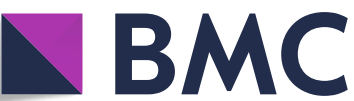

Part of Springer Nature (c) The Author(s). 2021 Open Access This article is licensed under a Creative Commons Attribution 4.0 International License, which permits use, sharing, adaptation, distribution and reproduction in any medium or format, as long as you give appropriate credit to the original author(s) and the source, provide a link to the Creative Commons licence, and indicate if changes were made. The images or other third party material in this article are included in the article's Creative Commons licence, unless indicated otherwise in a credit line to the material. If material is not included in the article's Creative Commons licence and your intended use is not permitted by statutory regulation or exceeds the permitted use, you will need to obtain permission directly from the copyright holder. To view a copy of this licence, visit http://creativecommons.org/licenses/by/4.0/. The Creative Commons Public Domain Dedication waiver (http://creativecommons.org/publicdomain/zero/1.0/) applies to the data made available in this article, unless otherwise stated in a credit line to the data. 


\section{Introduction}

Treatment of multiligament knee injury (MLKI) is still challenging for orthopedic surgeons because of its complexity and severity, and it might be more common than previously reported [1-6]. The incidence of MLKI might be underestimated because of spontaneous reductions and missed diagnoses due to combined injuries, such as vascular or nerve injury [4-7]. In cases of vascular emergencies, immediate vascular repair is necessary; thus, temporal stabilization is usually applied using an external fixator $[2,5]$.

However, in patients without any emergencies, the timing of surgery is still controversial [4, 8-10]. Early surgical reconstruction was advocated in some previous studies $[4,6,8]$, but others reported that early surgery resulted in stiffness, arthrofibrosis, and a reduced rate of return to work $[1,2,6,9,11,12]$. Even in patients with an anterior cruciate ligament (ACL) injury, the timing of surgery is also controversial [13-20]. Early ACL reconstruction is likely recommended [17, 21-23] because there is an increased risk of meniscus and cartilage injury if it is delayed [21, 22]. In a study of Norwegian National Registry data, the odds of a cartilage lesion increased by nearly $1 \%$ for each month from the injury date, and the odds of cartilage lesions were nearly twice as frequent when combined with meniscal tear [24]. However, there were also studies which reported no differences between early and delayed surgery $[13,18,25$, 26]. Thus, there is still a lack of consensus regarding the timing of surgery, regardless of the type of injury (single ACL injuries or MLKI) [6, 9, 16-20].

A paucity of evidence on which to base treatment decisions and the lack of consensus in ACL injuries further complicate the management of MLKI. Questions are established to determine if the decision-making parameters are similar for knees with a single ACL injury or those with MLKI and if they provide strong outcomes.

This systemic review including meta-analysis aimed to compare clinical outcomes and incidence of concomitant injuries in patients undergoing early vs delayed surgical treatment of single ACL injury and MLKI. We hypothesized that early surgery would result in better clinical outcomes and less incidence of concomitant injuries compared to delayed surgical treatment.

\section{Materials and methods}

\section{Protocol and registration}

This systematic review and meta-analysis were conducted according to the Preferred Reporting Items for Systematic Reviews and Meta-Analyses (PRISMA) guidelines and using the PRISMA checklist [27] and registered using the PROSPERO International prospective register of systematic reviews [28] (CRD42020145204).

\section{Search strategy}

A comprehensive literature search was conducted using several databases (PubMed, Embase, the Cochrane Library, the Cumulative Index to Nursing and Allied Health [CINAHL], and Scopus). In this study, the same included studies and some of the same extracted data were used in accordance with a previous meta-analysis comparing associated lesions in single ACL vs MLKIs. The date was restricted to all publications from the inception of these databases to April 30, 2020, and the search was conducted in May 2020. The search specifics were as follows: (Multiligament [All Fields] OR ("'multiple chronic conditions"[MeSH Terms] OR ("multiple"[All Fields] AND "chronic"[All Fields] AND "conditions"[All Fields] AND "acute"[All Fields]) OR "multiple chronic conditions"[All Fields] OR "multi"[All Fields]) AND ("ligaments"[MeSH Terms] OR "ligaments"[All Fields] OR "ligament"[All Fields]))) AND (“"meniscus" [MeSH Terms] OR "meniscus"[All Fields]) OR ("cartilage"[MeSH Terms] OR "cartilage"[All Fields])) AND ("knee"[MeSH Terms] OR "knee"[All Fields] OR "knee joint"[MeSH Terms] OR ("knee"[All Fields] AND “joint"[All Fields]) OR "knee joint"[All Fields]) AND ((“anterior cruciate ligament" [MeSH Terms] OR ("anterior"[All Fields] AND "cruciate"[All Fields] AND "ligament"[All Fields]) OR "anterior cruciate ligament"[All Fields] OR "acl”[All Fields]) OR "Anterior Cruciate Ligament"[Mesh] OR "anterior cruciate ligament"[All Fields]) AND AND ("chronic"[All Fields] AND "conditions"[All Fields] AND "acute"[All Fields]) AND ("1980/ 01/01"[PDAT]: “2020/04/30”[PDAT])) AND ((“meniscus" [MeSH Terms] OR "meniscus"[All Fields]) OR ("cartilage" [MeSH Terms] OR "cartilage"[All Fields])). The search criteria were broad to capture all potentially relevant articles, but only studies in English were included.

After combining the search results and removing duplicates, two authors independently screened the title and abstract for eligibility, and the agreement was assessed by kappa value. Subsequently, the same authors independently reviewed the full text of the selected studies. All references within the included studies were cross-referenced for inclusion if they were missed in the initial search. Systematic reviews and meta-analyses were excluded; however, their references were screened manually to find additional articles that were not identified in the first round. Disagreements were resolved by discussion between the two review authors or consultation with another author.

\section{Eligibility criteria}

Eligibility criteria for systemic review and meta-analysis were as follows: (1) English language, (2) level I to IV evidence, (3) publication between January 1980 and April 2020, (4) timing of the ligament reconstruction 
noted, and (5) "multiligament" defined as disruption of at least two of four major knee ligaments. The exclusion criteria were as follows: (1) not in the English language; (2) case report, clinical opinion, or technical note; (3) emergency treatment in MLKI; and (4) concomitant fracture around the knee (Fig. 1).

\section{Data extraction and collection}

The data extraction sheet was performed based on the checklist designed by Spindler et al. [29] and the consensus of authors for variables that should be reported. The following data were extracted: (1) study type, (2) level of evidence, (3) main purpose of the study, (4) number of cases, (5) age, (6) sex, (7) combined ligament injury, (8) concomitant injuries including the meniscus, (9) cartilage injuries, (10) reported complications, (11) timing of ACL reconstruction, (12) follow-up, (13) clinical outcomes, and (14) other relevant findings including revision. The data on the timing of surgery were recorded according to the definition of each study because of the heterogeneity of the included studies. The clinical outcomes were recorded as Lysholm scores, Tegner scores, International Knee Documentation Committee (IKDC) scores, number of meniscus tears, and number of cartilage injuries. The extracted data were also cross-checked for accuracy; any disagreements were settled by discussion between the two review authors or by consultation with another author.

\section{Grading of the quality of evidence}

The quality of evidence was evaluated by two authors using the guidelines of the Grading of Recommendations Assessment, Development and Evaluation (GRADE) working group [4]. The definitions of the grades of

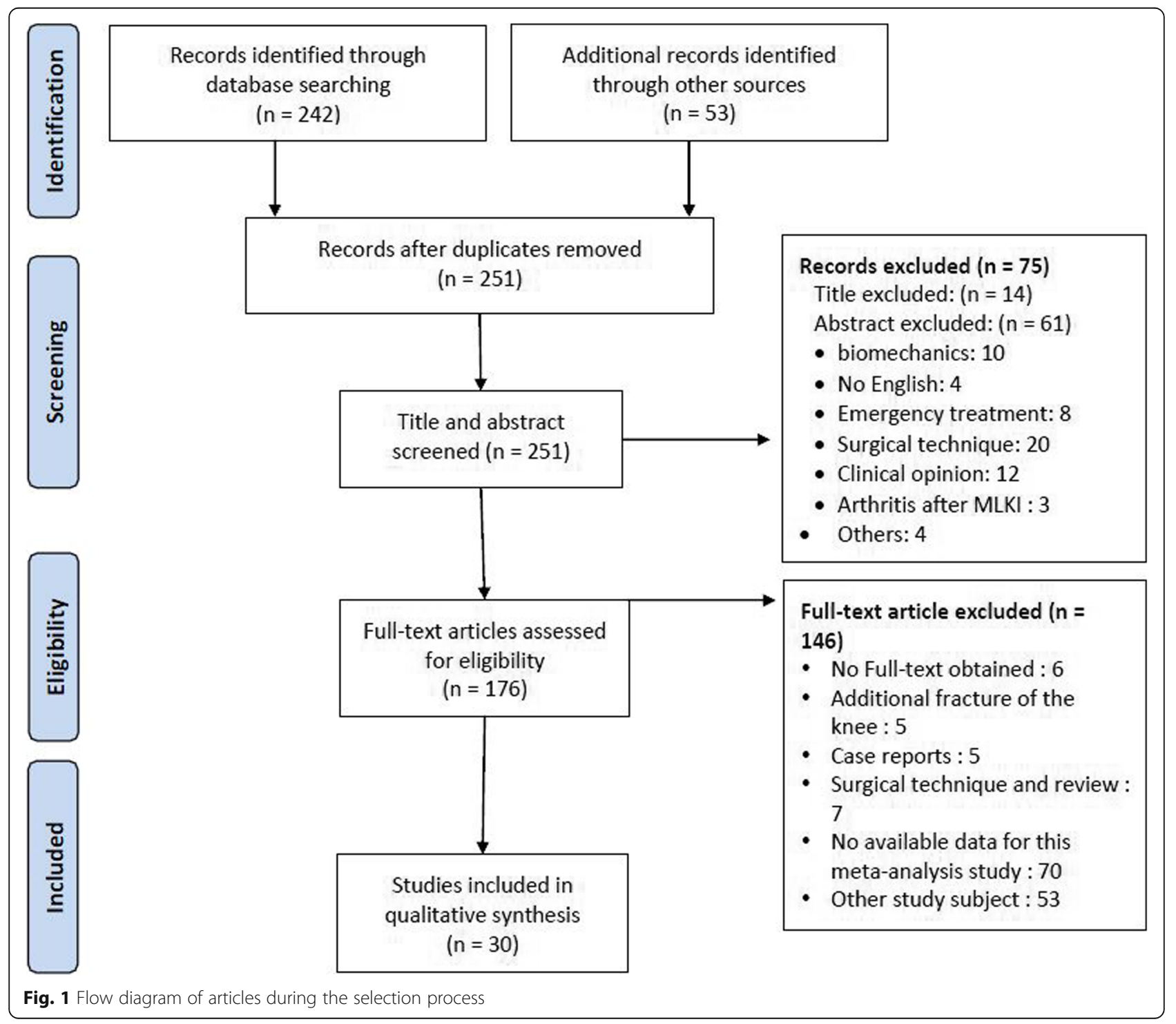


Table 1 Summary of the included studies

\begin{tabular}{|c|c|c|c|c|c|c|c|}
\hline Study name & Design & $\begin{array}{l}\text { Total no. of } \\
\text { patients } \\
\text { (criteria for } \\
\text { early vs } \\
\text { delayed) } \\
\end{array}$ & $\begin{array}{l}\text { Combined } \\
\text { ligament injury }\end{array}$ & $\begin{array}{l}\text { Combined meniscus } \\
\text { injury (positive/ } \\
\text { negative) }\end{array}$ & $\begin{array}{l}\text { Combined } \\
\text { cartilage } \\
\text { injury } \\
\text { (positive/ } \\
\text { negative) } \\
\end{array}$ & Clinical evaluation & $\begin{array}{l}\text { Overall } \\
\text { Follow- } \\
\text { up }\end{array}$ \\
\hline \multicolumn{8}{|c|}{ Isolated ACL injury } \\
\hline $\begin{array}{l}\text { Chen et al., } \\
2015[14, \\
46]\end{array}$ & $\begin{array}{l}\text { Retrospective } \\
\text { review } \\
\text { Cross-sectional } \\
\text { study }\end{array}$ & $\begin{array}{l}293 \\
\text { Early: } 160 \text { cases } \\
\text { Delayed: } 133 \\
\text { cases } \\
\text { (0-6 months vs } \\
\text { longer than } 7 \\
\text { months) }\end{array}$ & $\begin{array}{l}\text { Isolated ACL } \\
\text { injury }\end{array}$ & $\begin{array}{l}\text { Early: Total: } 86 \text { cases/ MM } \\
\text { tear: } 42 \text { cases/ LM tear: } \\
44 \text { cases } \\
\text { Delayed: Total: } 109 \\
\text { cases/MM tear: } 88 \text { cases/ } \\
\text { LM tear: } 21 \text { cases }\end{array}$ & $\begin{array}{l}\text { Early: Total: } 83 \\
\text { cases } \\
\text { Delayed: } \\
\text { Total: } 112 \\
\text { cases }\end{array}$ & Not reported & $\begin{array}{l}\text { Not } \\
\text { reported }\end{array}$ \\
\hline $\begin{array}{l}\text { Tandogan } \\
\text { et al., } 2004 \\
{[48]}\end{array}$ & Retrospective study & $\begin{array}{l}764 \\
\text { Early: } 510 \text { cases } \\
\text { Delayed: } 254 \\
\text { cases } \\
(0-12 \text { months } \\
\text { vs more than } \\
12 \text { months) }\end{array}$ & $\begin{array}{l}\text { Isolated ACL } \\
\text { injury }\end{array}$ & $\begin{array}{l}\text { Total meniscus tear: } 556 \\
\text { cases }\end{array}$ & $\begin{array}{l}\text { Early: } 31 \text { cases } \\
\text { Delayed: } 55 \\
\text { cases }\end{array}$ & Not reported & $\begin{array}{l}\text { Not } \\
\text { reported }\end{array}$ \\
\hline $\begin{array}{l}\text { Manandhar } \\
\text { et al., } 2018 \\
\text { [40] }\end{array}$ & Prospective study & $\begin{array}{l}104 \\
\text { Early: } 53 \text { cases } \\
\text { Delayed: } 51 \\
\text { cases } \\
\text { (within } 3 \text { weeks } \\
\text { vs after } 6 \\
\text { weeks) }\end{array}$ & $\begin{array}{l}\text { Isolated ACL } \\
\text { injury }\end{array}$ & $\begin{array}{l}\text { Early: Total: } 22 \text { cases / LM } \\
\text { tear: } 12 \text { cases / MM tear: } \\
6 \text { cases/ Both menisci } \\
\text { torn: } 4 \text { cases } \\
\text { Delayed: Total: } 34 \text { cases } \\
\text { /LM tear: } 8 \text { cases/ MM } \\
\text { tear: } 20 \text { cases/ Both } \\
\text { menisci torn: } 6 \text { cases }\end{array}$ & $\begin{array}{l}\text { Early: } 10 \text { cases } \\
\text { Delayed: } 28 \\
\text { cases }\end{array}$ & $\begin{array}{l}\text { IKDC Early: } 69.68 \pm \\
8.14 \text { vs delayed: } \\
67.14 \pm 6.08 \\
\text { Tegner Early } 4.15 \pm \\
1.45 \text { vs delayed: } \\
23.72 \pm 1.34\end{array}$ & $\begin{array}{l}\text { Not } \\
\text { reported } \\
\text { at least } \\
24 \text { weeks }\end{array}$ \\
\hline $\begin{array}{l}\text { Meighan } \\
\text { et al., } 2003 \\
{[42]}\end{array}$ & Retrospective study & $\begin{array}{l}31 \\
\text { Early: } 13 \text { cases } \\
\text { Delayed: } 18 \\
\text { cases } \\
\text { (within } 2 \text { weeks } \\
\text { vs between } 8 \\
\text { and } 12 \text { weeks) }\end{array}$ & $\begin{array}{l}\text { Isolated ACL } \\
\text { injury }\end{array}$ & $\begin{array}{l}\text { Early: Total: } 3 \text { cases } \\
\text { Delayed: Total: } 4 \text { cases }\end{array}$ & Not reported & Not reported & 1 year \\
\hline $\begin{array}{l}\text { Nikolic } \\
\text { et al., } 1998 \\
\text { [41] }\end{array}$ & Retrospective study & $\begin{array}{l}182 \\
\text { Early: } 66 \text { cases } \\
\text { Delayed: } 65 \\
\text { Excluded: } 51 \\
\text { cases } \\
\text { (not reported, } \\
\text { acute ACL vs } \\
\text { ACL-deficient } \\
\text { knee) }\end{array}$ & $\begin{array}{l}\text { Isolated ACL } \\
\text { injury }\end{array}$ & $\begin{array}{l}\text { Early: Total: } 51 \text { cases } \\
\text { Delayed: Total: } 53 \text { cases }\end{array}$ & Not reported & Not reported & $\begin{array}{l}\text { Not } \\
\text { reported }\end{array}$ \\
\hline $\begin{array}{l}\text { Raviraj } \\
\text { et al., } 2010 \\
{[25]}\end{array}$ & Retrospective study & $\begin{array}{l}99 \\
\text { Early: } 51 \text { cases } \\
\text { Delayed: } 48 \\
\text { cases } \\
\text { (<2 weeks vs } \\
\text { 4-6 weeks) }\end{array}$ & $\begin{array}{l}\text { Isolated ACL } \\
\text { injury }\end{array}$ & $\begin{array}{l}\text { Early: Total: } 38 \text { cases/MM } \\
\text { tear: } 18 \text { cases/ LM tear: } \\
20 \text { cases } \\
\text { Delayed: Total: } 35 \text { cases/ } \\
\text { MM tear: } 13 \text { cases/ LM } \\
\text { tear: } 22 \text { cases }\end{array}$ & $\begin{array}{l}\text { Early: } 29 \text { cases } \\
\text { Delayed: } 31 \\
\text { cases }\end{array}$ & $\begin{array}{l}\text { Lysholm score: early } \\
83.1 \text { ( } 80-90) \text { vs } \\
\text { delayed } 84.2 \text { ( } 82- \\
\text { 90) } \\
\text { Tegner activity } \\
\text { score: early } 6.1 \text { ( } 5 \text { to } \\
\text { 8) vs delayed } 5.9 \text { ( } 5 \\
\text { to 8) }\end{array}$ & $\begin{array}{l}32 \\
\text { months } \\
(26-36)\end{array}$ \\
\hline $\begin{array}{l}\text { Hur et al., } \\
2017 \text { [38] }\end{array}$ & Prospective study & $\begin{array}{l}91 \\
\text { Early: } 48 \text { cases } \\
\text { Delayed: } 43 \\
\text { cases } \\
\text { (within } 3 \text { weeks } \\
\text { vs more than } \\
3 \text { months) }\end{array}$ & $\begin{array}{l}\text { Isolated ACL } \\
\text { injury }\end{array}$ & $\begin{array}{l}\text { Early: Total: } 25 \text { cases/ MM } \\
\text { tear: } 14 \text { cases/ LM tear: } \\
15 \text { cases } \\
\text { Delayed: Total: } 27 \text { cases/ } \\
\text { MM tear: } 24 \text { cases/ LM } \\
\text { tear: } 9 \text { cases }\end{array}$ & $\begin{array}{l}\text { Early: } 15 \\
\text { cases, } \\
\text { Delayed: } 20 \\
\text { cases }\end{array}$ & $\begin{array}{l}\text { Lysholm: Early: } \\
94.5 \pm 8.9 \text { Delayed: } \\
96.3 \pm 3.7 \\
\text { Tegner: Early: } 6.0 \pm \\
1.6 \text { Delayed: } 5.6 \pm \\
1.5\end{array}$ & $\begin{array}{l}\text { Minimum } \\
2 \text { years }\end{array}$ \\
\hline $\begin{array}{l}\text { Li et al., } \\
2012 \text { [39] }\end{array}$ & Retrospective study & $\begin{array}{l}38 \\
\text { Early: } 17 \text { cases } \\
\text { Delayed: } 21 \\
\text { cases } \\
\text { ( }<3 \text { weeks } \\
\text { vs } \geq 3 \text { weeks) }\end{array}$ & $\begin{array}{l}\text { Isolated ACL } \\
\text { injury }\end{array}$ & $\begin{array}{l}\text { Early: Total: } 2 \text { cases } \\
\text { Delayed: Total: } 9 \text { cases }\end{array}$ & $\begin{array}{l}\text { Early: } 0 \text { cases, } \\
\text { Delayed: } 7 \\
\text { cases }\end{array}$ & $\begin{array}{l}\text { Lysholm: Early: } \\
94.7 \pm 9.3 \text { Delayed: } \\
92.2 \pm 7.8 \\
\text { Tegner: Early: } 6.6 \pm \\
1.9 \text { Delayed: } 6.3 \pm \\
1.3\end{array}$ & $\begin{array}{l}\text { Minimum } \\
2 \text { years }\end{array}$ \\
\hline
\end{tabular}


Table 1 Summary of the included studies (Continued)

\begin{tabular}{|c|c|c|c|c|c|c|c|}
\hline Study name & Design & $\begin{array}{l}\text { Total no. of } \\
\text { patients } \\
\text { (criteria for } \\
\text { early vs } \\
\text { delayed) } \\
\end{array}$ & $\begin{array}{l}\text { Combined } \\
\text { ligament injury }\end{array}$ & $\begin{array}{l}\text { Combined meniscus } \\
\text { injury (positive/ } \\
\text { negative) }\end{array}$ & $\begin{array}{l}\text { Combined } \\
\text { cartilage } \\
\text { injury } \\
\text { (positive/ } \\
\text { negative) } \\
\end{array}$ & Clinical evaluation & $\begin{array}{l}\text { Overall } \\
\text { Follow- } \\
\text { up }\end{array}$ \\
\hline $\begin{array}{l}\text { Ahlen and } \\
\text { Liden, } 2011 \\
\text { [43] }\end{array}$ & $\begin{array}{l}\text { Prospective cohort } \\
\text { study }\end{array}$ & $\begin{array}{l}61 \\
\text { Early: } 30 \text { cases } \\
\text { Delayed: } 31 \\
\text { cases } \\
\text { (within } 5 \\
\text { months vs } \\
\text { More than } 24 \\
\text { months) }\end{array}$ & $\begin{array}{l}\text { Isolated ACL } \\
\text { injury }\end{array}$ & $\begin{array}{l}\text { Early: Total: } 15 \text { cases/ MM } \\
\text { tear: } 4 \text { cases/ LM tear: } 9 \\
\text { cases } \\
\text { Delayed: Total: } 20 \text { cases/ } \\
\text { MM tear: } 14 \text { cases/ LM } \\
\text { tear: } 2 \text { cases }\end{array}$ & $\begin{array}{l}\text { Early: } 6 \text { cases } \\
\text { Delayed: } 9 \\
\text { cases }\end{array}$ & Not reported & $\begin{array}{l}25 \\
\text { months } \\
(18-43)\end{array}$ \\
\hline $\begin{array}{l}\text { Bottoni } \\
\text { et al., } 2008 \\
{[47]}\end{array}$ & $\begin{array}{l}\text { Prospective, } \\
\text { randomized cohort } \\
\text { study }\end{array}$ & $\begin{array}{l}70 \\
\text { Early: } 35 \text { cases } \\
\text { Delayed: } 35 \\
\text { cases } \\
\text { (within } 21 \text { days } \\
\text { vs beyond } 6 \\
\text { weeks) }\end{array}$ & $\begin{array}{l}\text { Isolated ACL } \\
\text { injury }\end{array}$ & $\begin{array}{l}\text { Early: Total: } 32 \text { cases/MM } \\
\text { tear: } 14 \text { cases/ LM tear: } \\
18 \text { cases } \\
\text { Delayed: Total: } 24 \text { cases/ } \\
\text { MM tear: } 15 \text { cases/LM } \\
\text { tear: } 9 \text { cases }\end{array}$ & $\begin{array}{l}\text { Early: } 9 \text { cases } \\
\text { Delayed: } 5 \\
\text { cases }\end{array}$ & $\begin{array}{l}\text { SANE: } 83.1 \text { vs } 81.4 \\
\text { Lysholm: } 80.6 \text { vs } \\
83.4 \\
\text { Tegner } 5.8 \text { vs } 4.9\end{array}$ & $\begin{array}{l}366 \text { days } \\
(185-869)\end{array}$ \\
\hline $\begin{array}{l}\text { Chen et al., } \\
2015[14, \\
46]\end{array}$ & $\begin{array}{l}\text { Prospective, } \\
\text { randomized cohort } \\
\text { study }\end{array}$ & $\begin{array}{l}55 \\
\text { Early: } 27 \text { cases } \\
\text { Delayed: } 28 \\
\text { cases } \\
\text { (3-7 weeks vs } \\
6-11 \text { months) }\end{array}$ & $\begin{array}{l}\text { Isolated ACL } \\
\text { injury }\end{array}$ & Not reported & Not reported & $\begin{array}{l}\text { Lysholm: } 47.26 \text { / } \\
93.37 \text { / } 95.04 \text { vs } 54.1 \\
\text { / } 91.64 \text { / } 92.64 \\
\text { Tegner: } 2.7 \text { / } 6.3 \text { / } \\
6.3 \text { vs } 2.5 \text { / } 6.1 \text { / } 6.3 \\
\text { IKDC: } 20 / 6 / 1 / 0 \text { vs } \\
\text { 17/9/2/0 }\end{array}$ & $\begin{array}{l}61 \\
\text { months }\end{array}$ \\
\hline $\begin{array}{l}\text { Cipolla } \\
\text { et al., } 1995 \\
{[16]}\end{array}$ & $\begin{array}{l}\text { Retrospective } \\
\text { review }\end{array}$ & $\begin{array}{l}770 \\
\text { Early: } 218 \text { cases } \\
\text { Delayed: } 552 \\
\text { cases } \\
\text { (within } 1 \text { week } \\
\text { vs later in } \\
\text { different } \\
\text { stages) }\end{array}$ & $\begin{array}{l}\text { Isolated ACL } \\
\text { injury }\end{array}$ & $\begin{array}{l}\text { Early: Total: } 63 \text { cases } \\
\text { Delayed: Total: } 412 \text { cases }\end{array}$ & Not reported & Not reported & $\begin{array}{l}\text { Not } \\
\text { reported }\end{array}$ \\
\hline $\begin{array}{l}\text { Frobell } \\
\text { et al., } 2010 \\
\text { [45] }\end{array}$ & $\begin{array}{l}\text { Prospective cohort } \\
\text { study }\end{array}$ & $\begin{array}{l}121 \\
\text { Early: } 62 \text { cases } \\
\text { Delayed: } 59 \\
\text { cases } \\
\text { (less than } 10 \\
\text { weeks vs more } \\
\text { than } 10 \text { weeks) }\end{array}$ & $\begin{array}{l}\text { Isolated ACL } \\
\text { injury }\end{array}$ & $\begin{array}{l}\text { Early: Total } 40 \text { cases } \\
\text { Chronic: Total } 50 \text { cases }\end{array}$ & Not reported & $\begin{array}{l}\text { KOOS subscale } \\
\text { - pain: } 87.2 \text { vs } 87.7 \\
\text { - symptoms: } 78.7 \text { vs } \\
83.0 \\
\text { - daily function: } 93.5 \\
\text { vs } 94.7 \\
\text { - sports function: } \\
\quad 71.8 \text { vs } 71.2 \\
\text { - QOL: } 67.3 \text { vs } 63.0 \\
\text { SF- } 36 \\
\text { - physical: } 82.1 \text { vs } \\
78.0 \\
\text { - mental: } 88.3 \text { vs } \\
83.8 \\
\text { Tegner: } 6.5 \text { vs } 5\end{array}$ & $\begin{array}{l}24.6 \\
\text { months } \\
(24.4- \\
24.7) \\
\text { vs } 25.0 \\
\text { months } \\
(24.7- \\
25.2)\end{array}$ \\
\hline $\begin{array}{l}\text { Frobell } \\
\text { et al., } 2013 \\
\text { [44] }\end{array}$ & $\begin{array}{l}\text { Prospective cohort } \\
\text { study (follow-up } \\
\text { study of Frobell } \\
\text { et al., } 2010 \text { [45]) }\end{array}$ & $\begin{array}{l}121 \\
\text { Early: } 61 \text { cases } \\
\text { Delayed: } 59 \\
\text { cases } \\
\text { (less than } 10 \\
\text { weeks vs more } \\
\text { than } 10 \text { weeks) }\end{array}$ & $\begin{array}{l}\text { Isolated ACL } \\
\text { injury }\end{array}$ & $\begin{array}{l}\text { Early: Total } 29 \text { cases } \\
\text { Delayed: Total } 32 \text { cases }\end{array}$ & Not reported & $\begin{array}{l}\text { KOOS: } 80 \text { vs } 82 \\
\text { KOOS subscale } \\
\text { - pain: } 91 \text { vs } 91 \\
\text { - symptoms: } 83 \text { vs } \\
87 \\
\text { - daily function: } 95 \\
\text { vs } 97 \\
\text { - sports function: } 76 \\
\text { vs } 79 \\
\text { - QOL: } 71 \text { vs } 69 \\
\text { SF-36 } \\
\text { - physical: } 85 \text { vs } 84 \\
\text { - mental: } 87 \text { vs } 85 \\
\text { Tegner: } 4 \text { vs } 4\end{array}$ & 5 years \\
\hline $\begin{array}{l}\text { Herbst } \\
\text { et al., } 2017\end{array}$ & $\begin{array}{l}\text { Prospective cohort } \\
\text { study }\end{array}$ & $\begin{array}{l}160 \\
\text { Early: } 51 \text { cases }\end{array}$ & $\begin{array}{l}\text { Isolated ACL } \\
\text { injury }\end{array}$ & $\begin{array}{l}\text { Early: MM tear: } 8 \text { cases/ } \\
\text { LM tear: } 14 \text { cases/ Both }\end{array}$ & Not reported & $\begin{array}{l}\text { Tegner (isolated } \\
\mathrm{ACL} \text { ): } 6.7 \pm 1.3 \mathrm{vs}\end{array}$ & $\begin{array}{l}24 \\
\text { months }\end{array}$ \\
\hline
\end{tabular}


Table 1 Summary of the included studies (Continued)

\begin{tabular}{|c|c|c|c|c|c|c|c|}
\hline Study name & Design & $\begin{array}{l}\text { Total no. of } \\
\text { patients } \\
\text { (criteria for } \\
\text { early vs } \\
\text { delayed) }\end{array}$ & $\begin{array}{l}\text { Combined } \\
\text { ligament injury }\end{array}$ & $\begin{array}{l}\text { Combined meniscus } \\
\text { injury (positive/ } \\
\text { negative) }\end{array}$ & $\begin{array}{l}\text { Combined } \\
\text { cartilage } \\
\text { injury } \\
\text { (positive/ } \\
\text { negative) } \\
\end{array}$ & Clinical evaluation & $\begin{array}{l}\text { Overall } \\
\text { Follow- } \\
\text { up }\end{array}$ \\
\hline [23] & & $\begin{array}{l}\text { Delayed: } 55 \\
\text { cases } \\
\text { (within } 48 \mathrm{~h} \text { vs } \\
\text { after } 6 \text { weeks) }\end{array}$ & & $\begin{array}{l}\text { menisci torn: } 8 \text { cases } \\
\text { Delayed: MM tear: } 13 \\
\text { cases/ LM tear: } 13 \text { cases/ } \\
\text { Both menisci torn: } 4 \\
\text { cases }\end{array}$ & & $\begin{array}{l}6.3 \pm 1.4 \\
\text { Tegner (with } \\
\text { meniscus injury) } \\
6.6 \pm 1.3 \text { vs } 6.3 \pm 1.5\end{array}$ & \\
\hline $\begin{array}{l}\text { Fok et al., } \\
2013 \text { [37] }\end{array}$ & $\begin{array}{l}\text { Retrospective } \\
\text { comparative study }\end{array}$ & $\begin{array}{l}150 \\
\text { Early: } 97 \text { cases } \\
\text { Delayed: } 53 \\
\text { cases } \\
\text { (less than } 12 \\
\text { months vs } \\
\text { more than } 12 \\
\text { months) }\end{array}$ & $\begin{array}{l}\text { Isolated ACL } \\
\text { injury }\end{array}$ & $\begin{array}{l}\text { Early: Total: } 58 \text { cases } \\
\text { Delayed: Total: } 41 \text { cases }\end{array}$ & $\begin{array}{l}\text { Early: } 40 \text { cases } \\
\text { Delayed: } 25 \\
\text { cases }\end{array}$ & $\begin{array}{l}\text { IKDC: with meniscal } \\
\text { injury } 60.4 \text { vs } \\
\text { without } 61.3 \\
\text { IKDC: with chondral } \\
\text { lesion } 60.1 \text { vs } \\
\text { without } 61.3 \\
\text { IKDC: Red-red tear } \\
56.7 \text { vs red-white } \\
\text { tear } 62.1 \text { vs white- } \\
\text { white tear } 60.1 \\
\text { Tegner score: preop } \\
\text { (<12 months) } 3.92 \\
\text { vs preop (> 12 } \\
\text { months } 3.41)\end{array}$ & $\begin{array}{l}\text { Not } \\
\text { reported }\end{array}$ \\
\hline \multicolumn{8}{|c|}{ Multiligament injuries } \\
\hline $\begin{array}{l}\text { Krych et al., } \\
2015 \text { [58] }\end{array}$ & Retrospective study & $\begin{array}{l}122 \\
\text { Early: } 62 \text { cases } \\
\text { Delayed: } 60 \\
\text { cases } \\
\text { (within } 3 \\
\text { months vs } \\
\text { after } 3 \text { months) }\end{array}$ & $\begin{array}{l}\text { KD I: } 25 \\
\text { KD II: } \\
\text { KD III: } 72 \\
\text { KD IV: } 16\end{array}$ & $\begin{array}{l}\text { Early: } 19 \text { cases } \\
\text { Delayed: } 21 \text { cases }\end{array}$ & $\begin{array}{l}\text { Total cartilage } \\
\text { injury } \\
52(48.0 \%) / \\
70(52.0 \%)\end{array}$ & Not reported & $\begin{array}{l}\text { Not } \\
\text { reported }\end{array}$ \\
\hline $\begin{array}{l}\text { Tardy et al., } \\
2017[56]\end{array}$ & Retrospective study & $\begin{array}{l}39 \\
\text { Early: } 22 \text { cases } \\
\text { Delayed: } 17 \\
\text { cases } \\
\text { ( } 7-30 \text { days vs } \\
\text { after } 3 \text { months) }\end{array}$ & $\begin{array}{l}\text { PMC vs PLC } \\
\text { injury }\end{array}$ & $\begin{array}{l}\text { Early: } 6 \text { cases } \\
\text { Delayed: } 7 \text { cases }\end{array}$ & Not reported & $\begin{array}{l}\text { Objective IKDC: } \\
\text { PMC 2A, 16B,1C vs } \\
\text { PLC 1A,13B,6C" } \\
\text { Subjective IKDC: } \\
\text { PMC } 81 \pm 15 \text { vs PLC } \\
70 \pm 17 " \\
\text { Lysholm } \\
\text { PMC } 89 \pm 7 \text { vs PLC } \\
79 \pm 11 \\
\text { Sports activity level } \\
\text { PMC: } 8 \text { at same } \\
\text { level /10 decreased } \\
\text { in activity level/1 } \\
\text { stopped sports } \\
\text { PLC: } 4 \text { at same } \\
\text { level/10 decreased } \\
\text { in activity level/ } 6 \\
\text { stopped sports }\end{array}$ & $\begin{array}{l}57 \\
\text { months } \\
(12-129)\end{array}$ \\
\hline $\begin{array}{l}\text { Moatshe } \\
\text { et al., } 2017 \\
\text { [59] }\end{array}$ & $\begin{array}{l}\text { Prospective cohort } \\
\text { study }\end{array}$ & $\begin{array}{l}65 \\
\text { Early: } 33 \text { cases } \\
\text { Delayed: } 32 \\
\text { cases } \\
\text { (less than } 21 \\
\text { days vs more } \\
\text { than } 21 \text { days) }\end{array}$ & $\begin{array}{l}\text { KD I: } 0 \\
\text { KD II: } 4 \\
\text { KD III: } 55 \\
\text { KD IV: } 6\end{array}$ & $25(38.5 \%) / 40(61.5 \%)$ & $\begin{array}{l}25(38.5 \%) / \\
40(61.5 \%)\end{array}$ & $\begin{array}{l}\text { Lysholm score } \\
\text { Early: } 86.9 \pm 15 \\
\text { Delayed: } 81 \pm 19 \\
\text { Tegner activity } \\
\text { Early: } 4 \pm 1.8 / \\
\text { Delayed: } 4 \pm 2 \\
\text { KOOS symptoms } 78 \\
\text { KOOS pain } 81 \\
\text { KOOS ADL } 87 \\
\text { KOOS sport } 54 \\
\text { KOOS QOL } 64 \\
\text { Single leg hop test } \\
\text { 88-93\% of the } \\
\text { uninjured leg }\end{array}$ & $\begin{array}{l}13.1 \text { years } \\
(10-18.8)\end{array}$ \\
\hline $\begin{array}{l}\text { Li et al., } \\
2013[51]\end{array}$ & Retrospective study & $\begin{array}{l}15 \\
\text { Early: } 6 \text { cases }\end{array}$ & $\begin{array}{l}\text { KD I: } 0 \\
\text { KD II: } 7\end{array}$ & Not reported & Not reported & $\begin{array}{l}\text { Lysholm score } \\
\text { Early: } 89.4 \pm 4.4\end{array}$ & $\begin{array}{l}7.5 \text { years } \\
(6-12)\end{array}$ \\
\hline
\end{tabular}


Table 1 Summary of the included studies (Continued)

\begin{tabular}{|c|c|c|c|c|c|c|c|}
\hline Study name & Design & $\begin{array}{l}\text { Total no. of } \\
\text { patients } \\
\text { (criteria for } \\
\text { early vs } \\
\text { delayed) }\end{array}$ & $\begin{array}{l}\text { Combined } \\
\text { ligament injury }\end{array}$ & $\begin{array}{l}\text { Combined meniscus } \\
\text { injury (positive/ } \\
\text { negative) }\end{array}$ & $\begin{array}{l}\text { Combined } \\
\text { cartilage } \\
\text { injury } \\
\text { (positive/ } \\
\text { negative) }\end{array}$ & Clinical evaluation & $\begin{array}{l}\text { Overall } \\
\text { Follow- } \\
\text { up }\end{array}$ \\
\hline & & $\begin{array}{l}\text { Delayed: } 9 \\
\text { cases } \\
\text { (<3 weeks vs } \\
\geq 3 \text { weeks) }\end{array}$ & $\begin{array}{l}\text { KD III: } 8 \\
\text { KD IV: } 0\end{array}$ & & & $\begin{array}{l}\text { Delayed: } 82.1 \pm 6.3 \\
\text { Tegner activity } \\
\text { Early: } 3.9 \text { / Delayed: } \\
3.4\end{array}$ & \\
\hline $\begin{array}{l}\text { Liow et al., } \\
2003 \text { [8] }\end{array}$ & Retrospective study & $\begin{array}{l}22 \\
\text { Early: } 8 \text { cases } \\
\text { Delayed: } 14 \\
\text { cases } \\
\text { (<2 weeks vs } \\
\geq 2 \text { weeks) }\end{array}$ & $\begin{array}{l}\text { KD I: } 7 \\
\text { KD II: } 2 \\
\text { KD III: } 11 \\
\text { KD IV: } 0\end{array}$ & Not reported & Not reported & $\begin{array}{l}\text { Lysholm score } \\
\text { Early: } 87(81-91) \\
\text { Delayed: } 75 \text { (53- } \\
\text { 100) } \\
\text { Tegner activity } \\
\text { Early: } 5 \text { / Delayed: } \\
\text { 4.4 }\end{array}$ & $\begin{array}{l}32 \\
\text { months } \\
(11-77)\end{array}$ \\
\hline $\begin{array}{l}\text { Noyes et al., } \\
1997 \text { [57] }\end{array}$ & Retrospective study & $\begin{array}{l}11 \\
\text { Early: } 7 \text { cases } \\
\text { Delayed: } 4 \\
\text { cases } \\
\text { (Early: mean } \\
14 \text { days } \\
\text { Delayed: mean } \\
22 \text { months) }\end{array}$ & $\begin{array}{l}\text { Both cruciate } \\
\text { ligaments torn: } \\
\text { 10, All ligaments } \\
\text { torn: } 1 \text { case }\end{array}$ & Not reported & $\begin{array}{l}\text { Early: } 0 \\
\text { Delayed: } 3\end{array}$ & Not reported & 4.5 years \\
\hline $\begin{array}{l}\text { Subbiah } \\
\text { et al., } 2011 \\
{[50]}\end{array}$ & Retrospective study & $\begin{array}{l}19 \\
\text { Early: } 11 \text { cases } \\
\text { Delayed: } 8 \\
\text { cases } \\
\text { (<3 weeks vs } \\
\geq 3 \text { weeks) }\end{array}$ & $\begin{array}{l}\text { KD I: } 5 \\
\text { KD II: } 3 \\
\text { KD III: } 11 \\
\text { KD IV: } 0\end{array}$ & $\begin{array}{l}\text { Total meniscus injury } \\
16(84 \%) / 3(16 \%)\end{array}$ & Not reported & $\begin{array}{l}\text { Lysholm score } \\
\text { Early: } 93.3 \pm 6.6 \\
\text { Delayed: } 90 \pm 5.8\end{array}$ & $\begin{array}{l}22 \\
\text { months } \\
(14-33)\end{array}$ \\
\hline $\begin{array}{l}\text { Wascher } \\
\text { et al., } 1999 \\
\text { [49] }\end{array}$ & Retrospective study & $\begin{array}{l}13 \\
\text { Early: } 9 \text { cases } \\
\text { Delayed: } 4 \\
\text { cases } \\
\text { (<3 weeks vs } \\
\geq 3 \text { weeks) }\end{array}$ & $\begin{array}{l}\text { ACL/PCL/MCL: } 7 \\
(53.8 \%) \\
\text { ACL/PCL/PLC: } 6 \\
(46.2 \%)\end{array}$ & $\begin{array}{l}\text { Early: Total: } 4 \text { cases } \\
\text { Delayed: Total: } 2 \text { cases }\end{array}$ & Not reported & $\begin{array}{l}\text { Lysholm score } \\
\text { Early: } 91.8 \pm 7.1 \\
\text { Delayed: } 79.3 \pm 22.7 \\
\text { IKDC: } 6 \text { nearly } \\
\text { normal }(46.2 \%) \\
\text { Meyers: } 11 \text { excellent } \\
\text { or good }(84.6 \%)\end{array}$ & $\begin{array}{l}38 \\
\text { months }\end{array}$ \\
\hline $\begin{array}{l}\text { Zhang } \\
\text { et al., } 2013 \\
\text { [52] }\end{array}$ & Retrospective study & $\begin{array}{l}59 \\
\text { Early: } 48 \text { cases } \\
\text { Delayed: } 11 \\
\text { cases } \\
\text { ( }<3 \text { weeks } \\
\text { vs }>3 \text { weeks) }\end{array}$ & $\begin{array}{l}\mathrm{ACL} / \mathrm{PCL} / \mathrm{MCL} / \\
\mathrm{PLC}\end{array}$ & Not reported & Not reported & $\begin{array}{l}\text { Lysholm score } \\
\text { Early: } 87.6 \pm 10.2 \\
\text { Delayed: } 80.5 \pm 13.3\end{array}$ & 2.5 years \\
\hline $\begin{array}{l}\text { Tzurbakis } \\
\text { et al., } 2006 \\
\text { [10] }\end{array}$ & Retrospective study & $\begin{array}{l}48 \\
\text { Early: } 38 \text { cases } \\
\text { Delayed: } 10 \\
\text { cases } \\
\text { (within } 3 \text { weeks } \\
\text { vs more than } \\
3 \text { weeks) }\end{array}$ & $\begin{array}{l}\text { Group A (ACL + } \\
\text { medial): } 12 \\
(25 \%) \\
\text { Group B } \\
\text { (cruciate + PLC): } \\
11(22.9 \%) \\
\text { Group C } \\
\text { (bicruciate + } \\
\text { collateral): } 25 \\
(52.1 \%)\end{array}$ & $\begin{array}{l}\text { Total meniscus tear } \\
\text { MM: } 20(41.7 \%) / 28 \\
(58.3 \%) \\
\text { LM: } 13(27.1 \%) / 35 \\
(72.9 \%)\end{array}$ & $\begin{array}{l}\text { Total cartilage } \\
\text { injury } \\
6(12.5 \%) / 42 \\
(87.5 \%)\end{array}$ & $\begin{array}{l}\text { Tegner: Early: } \\
4.4 \pm 2.1 \text { Delayed: } \\
5.2 \pm 2.2 \\
\text { Lysholm: Early: } 87 \pm \\
12.3 \text { Delayed: } \\
81.7 \pm 13.3\end{array}$ & $\begin{array}{l}51.3 \pm \\
29.9 \\
\text { months } \\
(24-96)\end{array}$ \\
\hline $\begin{array}{l}\text { Harner } \\
\text { et al., } 2004 \\
\text { [9] }\end{array}$ & $\begin{array}{l}\text { Retrospective } \\
\text { cohort study }\end{array}$ & $\begin{array}{l}31 \\
\text { Early: } 19 \text { cases } \\
\text { Delayed: } 12 \\
\text { cases } \\
\text { (within } 3 \text { weeks } \\
\text { vs more than } \\
3 \text { weeks) }\end{array}$ & $\begin{array}{l}\mathrm{ACL} / \mathrm{PCL} / \mathrm{MCL} / \\
\mathrm{PLC}\end{array}$ & Not reported & Not reported & $\begin{array}{l}\text { Lysholm: Early: } 91 \\
\text { Delayed: } 80 \\
\text { KOOS (daily): Early: } \\
91 \text { vs Delayed: } 84 \\
\text { KOOS (sports): Early: } \\
89 \text { vs Delayed: } 69 \\
\text { Meyers: Early: } 16 / 19 \\
\text { vs Delayed: } 7 / 12\end{array}$ & $\begin{array}{l}44 \mathrm{M} / \\
\text { minimum } \\
2 \mathrm{Y}\end{array}$ \\
\hline $\begin{array}{l}\text { Owens } \\
\text { et al., } 2007 \\
\text { [54] }\end{array}$ & Retrospective study & $\begin{array}{l}28 \\
\text { Early: } 20 \text { cases } \\
\text { Delayed: } 8\end{array}$ & $\begin{array}{l}\mathrm{ACL} / \mathrm{PCL} / \mathrm{MCL} / \\
\mathrm{PLC}\end{array}$ & $\begin{array}{l}\text { Total meniscus injury } \\
14(50 \%) / 14(50 \%)\end{array}$ & Not reported & $\begin{array}{l}\text { Lysholm score } \\
\text { Early: } 91.2 \pm 6.52 \\
\text { Delayed: } 83.6 \pm 7.3\end{array}$ & $\begin{array}{l}48 \\
\text { months } \\
\text { (13-82) }\end{array}$ \\
\hline
\end{tabular}


Table 1 Summary of the included studies (Continued)

\begin{tabular}{|c|c|c|c|c|c|c|c|}
\hline Study name & Design & $\begin{array}{l}\text { Total no. of } \\
\text { patients } \\
\text { (criteria for } \\
\text { early vs } \\
\text { delayed) }\end{array}$ & $\begin{array}{l}\text { Combined } \\
\text { ligament injury }\end{array}$ & $\begin{array}{l}\text { Combined meniscus } \\
\text { injury (positive/ } \\
\text { negative) }\end{array}$ & $\begin{array}{l}\text { Combined } \\
\text { cartilage } \\
\text { injury } \\
\text { (positive/ } \\
\text { negative) }\end{array}$ & Clinical evaluation & $\begin{array}{l}\text { Overall } \\
\text { Follow- } \\
\text { up }\end{array}$ \\
\hline & & $\begin{array}{l}\text { (within } 14 \text { days } \\
\text { vs greater than } \\
14 \text { days) }\end{array}$ & & & & & \\
\hline $\begin{array}{l}\text { Fanelli } \\
\text { et al., } 1996 \\
{[55]}\end{array}$ & Retrospective study & $\begin{array}{l}21 \\
\text { Early: } 13 \text { cases } \\
\text { Delayed: } 8 \\
\text { cases } \\
\text { ( } 2-4 \text { weeks vs } \\
6 \text { months-16 } \\
\text { years) }\end{array}$ & $\begin{array}{l}\text { All were } \mathrm{PCL} / \\
\mathrm{PLC} \text { injuries }\end{array}$ & Not reported & Not reported & $\begin{array}{l}\text { Lysholm score } \\
\text { Early: } 91.2 \text { / } \\
\text { Delayed: } 91.6 \\
\text { Tegner: Early } 5.2 \text { / } \\
\text { Delayed: } 5.0\end{array}$ & $\begin{array}{l}\text { Minimum } \\
24 \\
\text { months } \\
(24-54)\end{array}$ \\
\hline $\begin{array}{l}\text { Wajsfisz } \\
\text { et al., } 2014 \\
\text { [53] }\end{array}$ & Retrospective study & $\begin{array}{l}53 \\
\text { Early: } 10 \text { cases } \\
\text { Delayed: } 43 \\
\text { cases } \\
\text { (within } 21 \text { days } \\
\text { vs more than } \\
21 \text { days) }\end{array}$ & Not reported & Not reported & Not reported & $\begin{array}{l}\text { Lysholm score } \\
\text { Early: } 83 \text { / Delayed: } \\
76.5\end{array}$ & $\begin{array}{l}49 \\
\text { months } \\
(12-146)\end{array}$ \\
\hline
\end{tabular}

$A C L$ anterior cruciate ligament, $M M$ medial meniscus, $L M$ lateral meniscus, IKDC International Knee Documentation Committee, KOOS Knee Injury and Osteoarthritis Outcome Score, $Q O L$ quality of life, KD Schenck knee dislocation type, $P M C$ posteromedial corner, $P L C$ posterolateral corner, $A D L$ activities of daily living, $P C L$ posterior cruciate ligament, $M C L$ medial collateral ligament

evidence were as follows: (1) high, when further research is unlikely to change confidence in the estimate of the effect; (2) moderate, when further research is likely to have an important impact on the confidence in the estimate of the effect and may change it; (3) low, when further research is particularly likely to have an important impact on the confidence in the estimate of the effect and is likely to change it; and (4) very low, when any estimate of the effect is extremely uncertain [4]. Disagreements were resolved by discussion and assessed by kappa value.

\section{Assessment of methodological quality}

Two investigators independently assessed the methodological quality of each study using the Downs and Black quality assessment tool [30], which was developed for use in systemic reviews of both randomized and nonrandomized studies. This tool consists of 27 questions that assess the criteria for reporting, external validity, and internal validity (measurement and confounding). The highest possible score is 32 . Disagreements were resolved by discussion and assessed by kappa value. For the additional graphical assessment of the risk of bias across the studies, ROBINS-I (Risk Of Bias In Nonrandomized Studies of Interventions) [31, 32] was also used, which was released by the Cochrane NonRandomized Study Group recently.

The possibility of publication bias was examined by Egger's test based on Galbraith plots [33] with a random-effects model. Funnel plot asymmetry and Egger's tests were conducted to examine the possibility of publication bias. Moreover, the trim-and-fill method and calculation of a fail-safe number were also performed to evaluate the robustness of publication bias $[34,35]$.

\section{Statistical analysis}

Data analyses were performed with Review Manager software (version 5.3; Nordic Cochrane Centre, the Cochrane Collaboration) and the $\mathrm{R}$ program (version 3.5.3, the R Foundation) using the "meta" and "metafor" packages. Statistical heterogeneity was assessed with $I^{2}$ statistics: $I^{2}>50 \%$, substantial heterogeneity; $20 \%<I^{2} \leq$ $50 \%$, moderate heterogeneity; $I^{2}<20 \%$, low heterogeneity. A random-effects model was used to analyze the more robust results. Forest plots were used to show the outcome, pooled estimate of effect, and overall summary effect of each study. The treatment effects were measured by $95 \%$ confidence intervals (CIs) if the outcomes were measured on the same scale. Because of the high risk of bias due to the low level of evidence studies, the pooled mean differences were not used in clinical outcomes (Lysholm and Tegner scores). The mean synthesis and non-weighted mean differences (NMDs) of the bestevidence synthesis method were used instead to evaluate the clinical outcomes [36]. Comparisons between early and delayed values from each study were made using two-sample Z-tests using a $p$ value $<0.05$ (http://www. statskingdom.com/120MeanNormal2.html). The pooled odds ratio (OR) for the forest plot was also measured if the outcomes were collected as categorical data using the Mantel-Haenszel method. The heterogeneity of the 
Table 2 Quality assessment of included studies for meta-analysis

\begin{tabular}{|c|c|c|c|c|c|c|c|c|}
\hline Study name & Design & $\begin{array}{l}\text { Total no. of } \\
\text { patients }\end{array}$ & $\begin{array}{l}\text { Reporting } \\
\text { (11) }\end{array}$ & $\begin{array}{l}\text { External } \\
\text { validity }\end{array}$ & $\begin{array}{l}\text { Internal } \\
\text { validity: bias }\end{array}$ & $\begin{array}{l}\text { Internal validity: } \\
\text { confounding (selection } \\
\text { bias) }\end{array}$ & Power & Total \\
\hline \multicolumn{9}{|l|}{ Isolated ACL injury } \\
\hline $\begin{array}{l}\text { Chen et al., } 2015 \\
{[14,46]}\end{array}$ & Retrospective study & 293 & 5 & 1 & 3 & 3 & 5 & 17 \\
\hline $\begin{array}{l}\text { Tandogan et al., } \\
2004 \text { [48] }\end{array}$ & Retrospective study & 764 & 6 & 1 & 3 & 2 & 5 & 17 \\
\hline $\begin{array}{l}\text { Manandhar et al., } \\
2018 \text { [40] }\end{array}$ & $\begin{array}{l}\text { Prospective cohort } \\
\text { study }\end{array}$ & 104 & 9 & 1 & 5 & 3 & 5 & 23 \\
\hline $\begin{array}{l}\text { Meighan et al., } \\
2003 \text { [42] }\end{array}$ & Retrospective study & 31 & 7 & 1 & 3 & 3 & 2 & 16 \\
\hline $\begin{array}{l}\text { Nikolic et al., } 1998 \\
\text { [41] }\end{array}$ & Retrospective study & 182 & 7 & 1 & 3 & 2 & 5 & 18 \\
\hline $\begin{array}{l}\text { Raviraj et al., } 2010 \\
\text { [25] }\end{array}$ & Retrospective study & 99 & 8 & 1 & 3 & 3 & 4 & 19 \\
\hline $\begin{array}{l}\text { Hur et al., } 2017 \\
\text { [38] }\end{array}$ & Prospective study & 91 & 8 & 1 & 3 & 4 & 5 & 21 \\
\hline Li et al., 2012 [39] & Retrospective study & 38 & 7 & 1 & 3 & 3 & 4 & 18 \\
\hline $\begin{array}{l}\text { Ahlen and Liden } \\
\text { et al., } 2011 \text { [43] }\end{array}$ & $\begin{array}{l}\text { Prospective cohort } \\
\text { study }\end{array}$ & 61 & 8 & 1 & 3 & 3 & 5 & 20 \\
\hline $\begin{array}{l}\text { Bottoni et al., } 2008 \\
\text { [47] }\end{array}$ & $\begin{array}{l}\text { Prospective, } \\
\text { randomized study }\end{array}$ & 70 & 10 & 1 & 4 & 6 & 4 & 25 \\
\hline $\begin{array}{l}\text { Chen et al., } 2015 \\
{[14,46]}\end{array}$ & $\begin{array}{l}\text { Prospective, } \\
\text { randomized study }\end{array}$ & 55 & 8 & 1 & 4 & 4 & 3 & 20 \\
\hline $\begin{array}{l}\text { Cipolla et al., } 1995 \\
\text { [16] }\end{array}$ & Retrospective study & 1103 & 3 & 1 & 2 & 2 & 5 & 13 \\
\hline $\begin{array}{l}\text { Frobell et al., } 2010 \\
\text { [45] }\end{array}$ & $\begin{array}{l}\text { Prospective cohort } \\
\text { study }\end{array}$ & 121 & 10 & 1 & 5 & 4 & 5 & 25 \\
\hline $\begin{array}{l}\text { Frobell et al., } 2013 \\
\text { [44] }\end{array}$ & $\begin{array}{l}\text { Prospective cohort } \\
\text { study }\end{array}$ & 121 & 10 & 1 & 5 & 4 & 5 & 25 \\
\hline $\begin{array}{l}\text { Herbst et al., } 2017 \\
\text { [23] }\end{array}$ & $\begin{array}{l}\text { Prospective cohort } \\
\text { study }\end{array}$ & 160 & 8 & 1 & 5 & 3 & 5 & 22 \\
\hline Fok et al., 2013 [37] & $\begin{array}{l}\text { Retrospective } \\
\text { comparative study }\end{array}$ & 150 & 10 & 1 & 3 & 2 & 4 & 20 \\
\hline \multicolumn{9}{|l|}{ Multiligament injuries } \\
\hline $\begin{array}{l}\text { Krych et al., } 2015 \\
\text { [58] }\end{array}$ & Retrospective study & 122 & 3 & 1 & 3 & 3 & 5 & 15 \\
\hline $\begin{array}{l}\text { Tardy et al., } 2017 \\
\text { [56] }\end{array}$ & Retrospective study & 39 & 4 & 1 & 3 & 3 & 2 & 13 \\
\hline $\begin{array}{l}\text { Moatshe et al., } \\
2017 \text { [59] }\end{array}$ & $\begin{array}{l}\text { Prospective cohort } \\
\text { study }\end{array}$ & 65 & 9 & 1 & 4 & 3 & 4 & 21 \\
\hline Li et al., 2013 [51] & Retrospective study & 15 & 4 & 1 & 3 & 3 & 0 & 11 \\
\hline $\begin{array}{l}\text { Liow et al., } 2003 \\
\text { [8] }\end{array}$ & Retrospective study & 22 & 5 & 1 & 3 & 3 & 1 & 13 \\
\hline $\begin{array}{l}\text { Subbiah et al., } \\
2011 \text { [50] }\end{array}$ & Retrospective study & 19 & 7 & 1 & 3 & 2 & 0 & 13 \\
\hline $\begin{array}{l}\text { Zhang et al., } 2013 \\
\text { [52] }\end{array}$ & Retrospective study & 59 & 7 & 1 & 3 & 2 & 3 & 16 \\
\hline $\begin{array}{l}\text { Tzurbakis et al., } \\
2006 \text { [10] }\end{array}$ & Retrospective study & 48 & 5 & 1 & 2 & 0 & 3 & 11 \\
\hline $\begin{array}{l}\text { Noyes, et al., } 1997 \\
\text { [57] }\end{array}$ & Retrospective study & 11 & 3 & 1 & 2 & 0 & 0 & 6 \\
\hline Harner et al., 2004 & Retrospective study & 31 & 9 & 1 & 3 & 3 & 2 & 18 \\
\hline
\end{tabular}


Table 2 Quality assessment of included studies for meta-analysis (Continued)

\begin{tabular}{|c|c|c|c|c|c|c|c|c|}
\hline Study name & Design & $\begin{array}{l}\text { Total no. of } \\
\text { patients }\end{array}$ & $\begin{array}{l}\text { Reporting } \\
\text { (11) }\end{array}$ & $\begin{array}{l}\text { External } \\
\text { validity }\end{array}$ & $\begin{array}{l}\text { Internal } \\
\text { validity: bias }\end{array}$ & $\begin{array}{l}\text { Internal validity: } \\
\text { confounding (selection } \\
\text { bias) }\end{array}$ & Power & Total \\
\hline \multicolumn{9}{|l|}{ [9] } \\
\hline $\begin{array}{l}\text { Owens et al., } 2007 \\
\text { [54] }\end{array}$ & Retrospective study & 28 & 4 & 1 & 2 & 0 & 1 & 8 \\
\hline $\begin{array}{l}\text { Wascher et al., } \\
1999 \text { [49] }\end{array}$ & Retrospective study & 13 & 5 & 1 & 2 & 0 & 0 & 8 \\
\hline $\begin{array}{l}\text { Fanelli et al., } 1996 \\
\text { [55] }\end{array}$ & Retrospective study & 21 & 3 & 0 & 1 & 0 & 1 & 5 \\
\hline $\begin{array}{l}\text { Wajsfisz et al., } 2014 \\
\text { [53] }\end{array}$ & Retrospective study & 53 & 4 & 0 & 0 & 0 & 0 & 4 \\
\hline
\end{tabular}

binary categorical data was also evaluated using the L'Abbé plot. The subgroup analysis was performed according to the single ACL injury and MLKI groups, and meta-regression analyses with a mixed-effects model were also performed to assess the effects of the potential moderators (single ACL injury vs MLKI/prospective vs retrospective studies) on the overall heterogeneity if significant heterogeneity was observed.

The inter-rater reliability was assessed using kappa statistics ( $\mathrm{k}$ ) to determine the degree of agreement in the study selection and risk assessment. Agreement was deemed fair $(\kappa=0.21-0.40)$, moderate $(\kappa=0.41-0.60)$, substantial $(\mathrm{K}=0.61-0.80)$, or almost perfect $(\mathrm{K}=0.81-$ 1.00 ). In all analyses, a $p$ value $<0.05$ was considered significant with a two-sided tail.

\section{Results}

\section{Study characteristics}

The selection process for the studies is shown in the flow diagram of Fig. 1. Sixteen studies [14, 16, 23, 25, 37-48] on single ACL injury (3004 patients) and 14 studies on MLKI [30, 32, 33, 49-59] (545 patients) were included in this meta-analysis of early vs delayed surgery. Details of these included studies are presented in Table 1.

\section{Assessment of methodological quality}

The results of the quality assessment in the included studies are shown in Table $2(\kappa=0.73$, substantial agreement). The overall bar plot of the ROBINS-I tool is summarized in Fig. 2 and Additional file 1: Figure S1 ( $\mathrm{K}=$ 0.83 , almost perfect agreement).

A funnel plot was used to evaluate the scores which could be obtained by weighted values (total meniscus tears and cartilage injuries). The funnel plot suggested a publication bias in the assessment of meniscus tear; the trim-and-fill method and calculation of fail-safe number were then performed to further assess the publication bias.

In the analysis of meniscus tear, evidence of asymmetry was observed (Fig. 3a), and this result was further supported by an analysis using Egger's test $(p=0.000)$. The adjusted funnel plot after the trim-and-fill method (Fig. 3b) indicated the absence of publication bias with eight added studies, but the observed outcome was changed to reinforce the direction of the outcome (before vs after trim and fill, OR 1.73 vs 3.42). Moreover, the fail-safe number was calculated by the Rosenthal approach [34] as $227(p<0.0001)$, which is a robust result for publication bias for this study.

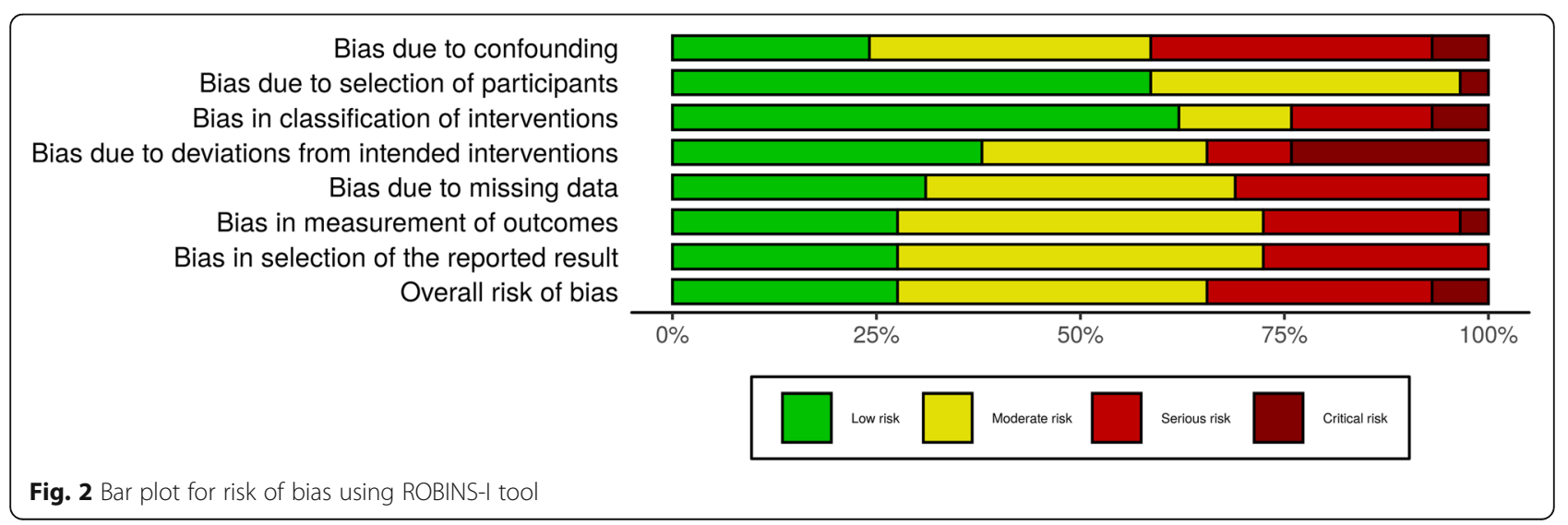




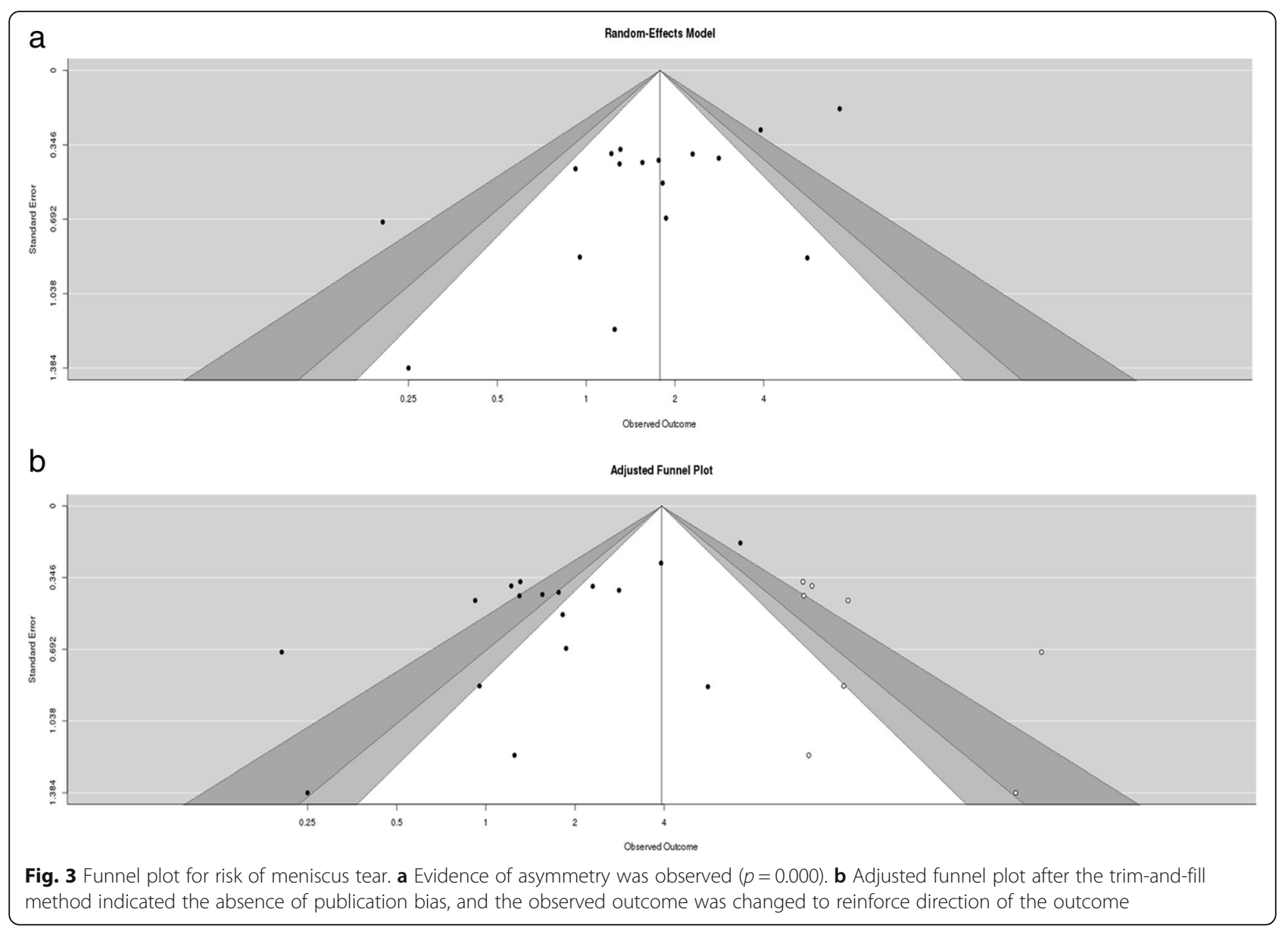

In the analysis of cartilage injury, there was no evidence of publication bias $(p=0.618$, Additional file 2 : Figure S2). The fail-safe number was calculated as 168 for the cartilage injury $(p<0.0001)$. The cartilage injury analysis showed a robust result for publication bias.

\section{GRADE evidence quality of each outcome}

The GRADE evidence quality of each outcome is presented in Table 3 ( $\kappa=0.65$, substantial agreement). Four outcomes were separately evaluated: one of very low quality and three of low quality. The overall results were found to have a trend of increased risk of meniscus tear and cartilage injury and decreased Lysholm and Tegner scores in the delayed surgery group. However, almost all outcomes had limitations in study design, imprecise data, and directness of the studies.

\section{Risk of concomitant meniscus tear}

The number of total meniscus injuries according to early vs delayed surgery was determined in 13 studies (2064 patients) on single ACL injury [23, 25, 37-44, 46, 47] and 4 studies (185 patients) on MLKI [49, 56-58]. The overall risk of meniscus tear in the delayed surgery group was significantly higher than that in the early surgery group, but this showed substantial heterogeneity (OR 1.73 [95\% CI 1.1-2.73], $p=0.01 ; I^{2}=78 \%, p<0.01$ ), as did the L'Abbé plot (Fig. 4a, Additional file 3: Figure S3). In the subgroup analysis according to single ACL injury and MLKI, the risk of meniscus tear in delayed surgery of single ACL injury was significantly higher than that in early surgery with substantial heterogeneity (OR 1.88 [95\% CI 1.13-3.13], $p=0.015 ; I^{2}=81 \%, p<$ 0.01 ), but the risk of meniscus tear in MLKI was not significant regardless of the timing of surgery (early or delayed) (OR 1.23 [95\% CI 0.66-2.28], $p=0.512 ; I^{2}=0 \%$, $p=0.64)$ (Fig. 4a).

The result of the meta-regression analysis indicated that single ACL injury and MLKI/prospective and retrospective design were not significant moderators of overall heterogeneity (Fig. 4b, $p=0.255$ ).

\section{Risk of concomitant cartilage injury}

The number of cartilage injuries according to the timing of surgery (early vs delayed) was described in 10 studies (1681 patients) regardless of single ACL injury or MLKI [25, 37-40,43, 46-48, 57]. Only one study [57] on MLKI reported cartilage injury result according to the timing of surgery (early vs delayed); thus, the subgroup 
Table 3 GRADE evidence quality for each outcome

\begin{tabular}{|c|c|c|c|c|c|c|c|c|c|}
\hline \multicolumn{6}{|c|}{ Quality assessment } & \multicolumn{4}{|c|}{ Summary of findings } \\
\hline \multirow{2}{*}{$\begin{array}{l}\text { Number } \\
\text { of } \\
\text { studies }\end{array}$} & \multirow[t]{2}{*}{ Design } & \multirow[t]{2}{*}{ Quality } & \multirow[t]{2}{*}{ Consistency } & \multirow[t]{2}{*}{ Directness } & \multirow{2}{*}{$\begin{array}{l}\text { Other } \\
\text { modifying } \\
\text { factors }\end{array}$} & \multicolumn{2}{|l|}{$\begin{array}{l}\text { No. of } \\
\text { patients }\end{array}$} & \multirow[t]{2}{*}{ Summary } & \multirow[t]{2}{*}{ Quality } \\
\hline & & & & & & Delayed & Early & & \\
\hline \multicolumn{10}{|c|}{ Concomitant meniscus tear } \\
\hline 18 & $\begin{array}{l}\text { RCT: } 7 \\
\text { Non- } \\
\text { RCT: } 11\end{array}$ & $\begin{array}{l}\text { Very } \\
\text { serious } \\
\text { limitations } \\
(-2)\end{array}$ & $\begin{array}{l}\text { Important } \\
\text { inconsistency } \\
(-1)\end{array}$ & $\begin{array}{l}\text { Some } \\
\text { uncertainty } \\
(-1)\end{array}$ & $\begin{array}{l}\text { Evidence of a } \\
\text { dose- } \\
\text { response gra- } \\
\text { dient }(+1)\end{array}$ & 1308 & 1062 & $\begin{array}{l}\text { The incidence of meniscus tear in delayed } \\
\text { group was higher than in early group. } \\
\text { Only } 3 \text { studies reported higher incidence } \\
\text { of meniscus tear in early group }\end{array}$ & Low \\
\hline \multicolumn{10}{|c|}{ Lysholm score } \\
\hline 17 & $\begin{array}{l}\text { RCT: } 4 \\
\text { Non- } \\
\text { RCT: } 13\end{array}$ & $\begin{array}{l}\text { Very } \\
\text { serious } \\
\text { limitations } \\
(-2)\end{array}$ & $\begin{array}{l}\text { No important } \\
\text { inconsistency }\end{array}$ & $\begin{array}{l}\text { Some } \\
\text { uncertainty } \\
(-1)\end{array}$ & $\begin{array}{l}\text { Imprecise or } \\
\text { sparse data } \\
(-1) \text {. } \\
\text { Evidence of a } \\
\text { dose- } \\
\text { response gra- } \\
\text { dient }(+1)\end{array}$ & 402 & 455 & $\begin{array}{l}\text { The Lysholm scores decreased in delayed } \\
\text { surgery group. Only } 2 \text { studies reported } \\
\text { higher scores in delayed surgery group }\end{array}$ & Low \\
\hline \multicolumn{10}{|c|}{ Tegner score } \\
\hline 15 & $\begin{array}{l}\text { RCT: } 8 \\
\text { Non- } \\
\text { RCT: } 7\end{array}$ & $\begin{array}{l}\text { Very } \\
\text { serious } \\
\text { limitations } \\
(-2)\end{array}$ & $\begin{array}{l}\text { No important } \\
\text { inconsistency }\end{array}$ & $\begin{array}{l}\text { Some } \\
\text { uncertainty } \\
(-1)\end{array}$ & None & 496 & 524 & $\begin{array}{l}\text { The Tegner scores decreased in delayed } \\
\text { surgery group, but those for delayed } \\
\text { surgery group in MLKI were marginal }\end{array}$ & Low \\
\hline \multicolumn{10}{|c|}{ Concomitant cartilage injury } \\
\hline 10 & $\begin{array}{l}\text { RCT: } 5 \\
\text { Non- } \\
\text { RCT: } 5\end{array}$ & $\begin{array}{l}\text { Very } \\
\text { serious } \\
\text { limitations } \\
(-2)\end{array}$ & $\begin{array}{l}\text { Important } \\
\text { inconsistency } \\
(-1)\end{array}$ & $\begin{array}{l}\text { Some } \\
\text { uncertainty } \\
(-1)\end{array}$ & $\begin{array}{l}\text { Imprecise or } \\
\text { sparse data } \\
(-1)\end{array}$ & 673 & 1008 & $\begin{array}{l}\text { The incidence of cartilage injury in delayed } \\
\text { group was higher than in early group. } \\
\text { Only } 1 \text { study of MLKI was included for this } \\
\text { meta-analysis. }\end{array}$ & $\begin{array}{l}\text { Very } \\
\text { low }\end{array}$ \\
\hline
\end{tabular}

GRADE Grading of Recommendations Assessment, Development and Evaluation, $R C T$ randomized controlled trial, MLKI multiligament knee injury

analysis was not performed. The overall risk of cartilage injury in the delayed surgery group was significantly higher than that in the early surgery group, but this showed substantial heterogeneity (OR 2.48 [95\% CI $\left.1.46-4.2], \quad p=0.0007 ; I^{2}=70 \%, p<0.01\right)$, as did the L'Abbé plot (Fig. 5a, Additional file 4: Figure S4).

The result of the meta-regression analysis indicated that prospective and retrospective design were not significant moderators of overall heterogeneity (Fig. 5b, $p=$ $0.336)$.

\section{Lysholm score}

The Lysholm scores according to the timing of surgery (early vs delayed) were described in 6 studies (444 patients) on single ACL injury $[23,25,38,39,43]$ and 11 studies (413 patients) on MLKI [30, 32, 33, 49-55, 59]. The overall Lysholm scores in the delayed surgery group were lower than those in the early surgery group (early vs delayed, $89.9 \pm 3.64$ vs $85.3 \pm 5.9 ; p<0.001$; NMD 5.3 [ $95 \%$ CI -7.37 to -3.23$]$ ) (Fig. 6). In the subgroup analysis according to the type of injury (single ACL injury and MLKI), the Lysholm score of the delayed surgery MLKI group was significantly lower than that of the early surgery MLKI group (early vs delayed, $89.0 \pm 2.87$ vs $82.8 \pm 4.61 ; p<0.001$; NMD -7.1 [95\% CI -9.24 to 4.96]), and the Lysholm score of the delayed surgery single ACL group was also significantly lower than that of the early surgery single ACL group (early vs delayed, $91.7 \pm 4.21$ vs $89.8 \pm 5.34 ; p<0.001 ; \mathrm{NMD}-1.95[95 \%$ CI -4.78 to 0.88$]$ ).

\section{Tegner score}

The Tegner scores according to the timing of surgery (early vs delayed) were described in 9 studies (728 patients) on single ACL $[23,25,38-40,43,44]$ and five studies (171 patients) on MLKI [30, 33, 51, 55, 59]. In the study by Herbst et al. [23], the researchers reported the results as separate groups according to the meniscus injury; thus, we analyzed the results as two different studies. The overall Tegner scores in the delayed surgery group were significantly decreased (early vs delayed, $5.4 \pm 1.05$ vs $5.1 \pm 1.01 ; p<0.001$; NMD -0.25 [95\% CI -0.45 to -0.05$]$ ) compared to those in the early surgery group (Fig. 7). In the subgroup analysis according to the type of injury (single ACL injury and MLKI), the Tegner score of the delayed surgery single ACL injury group was significantly lower than that of the early surgery single ACL injury group (early vs delayed, $5.8 \pm 0.97$ vs $5.5 \pm 0.97 ; p<0.001 ; \mathrm{NMD}-0.3$ [95\% CI -0.51 to $0.15]$ ), but the Tegner score in the MLKI group was not significant, regardless of the timing of surgery (early vs 


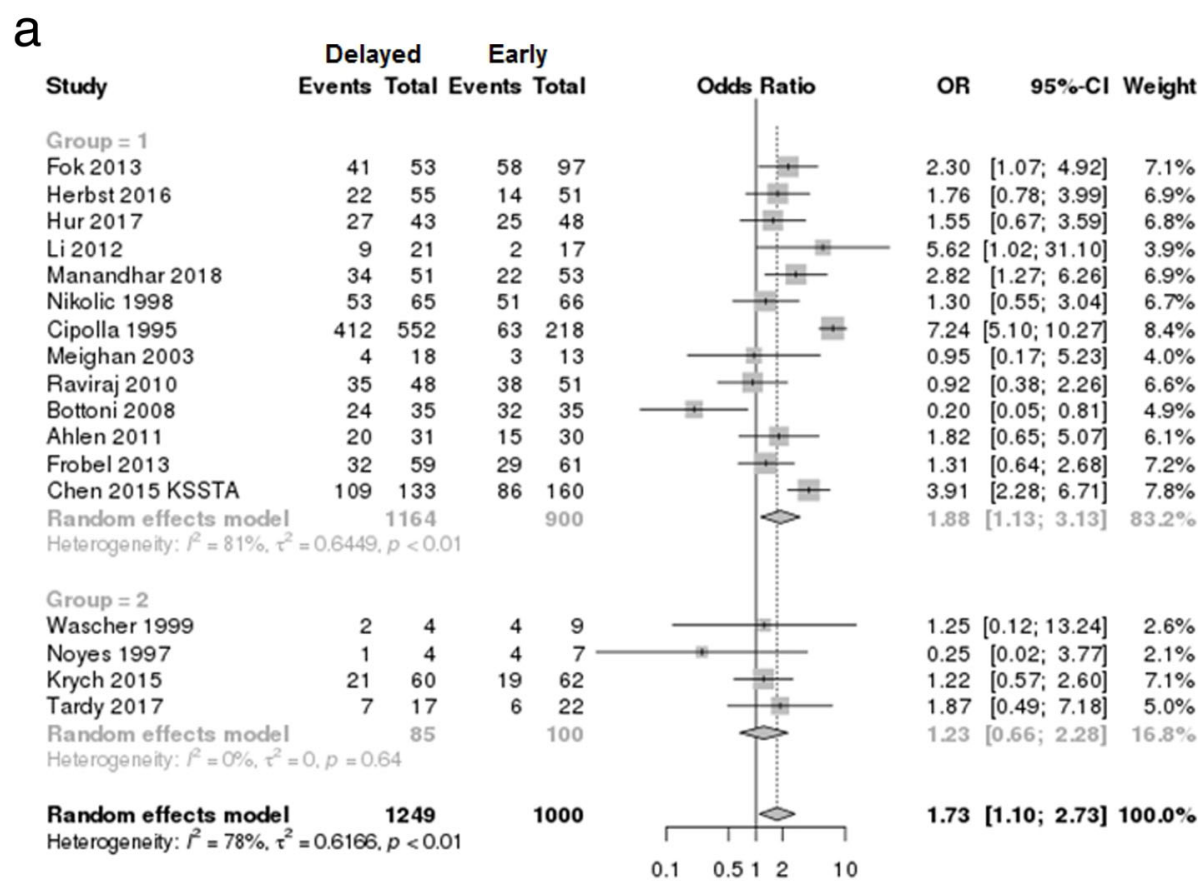

b

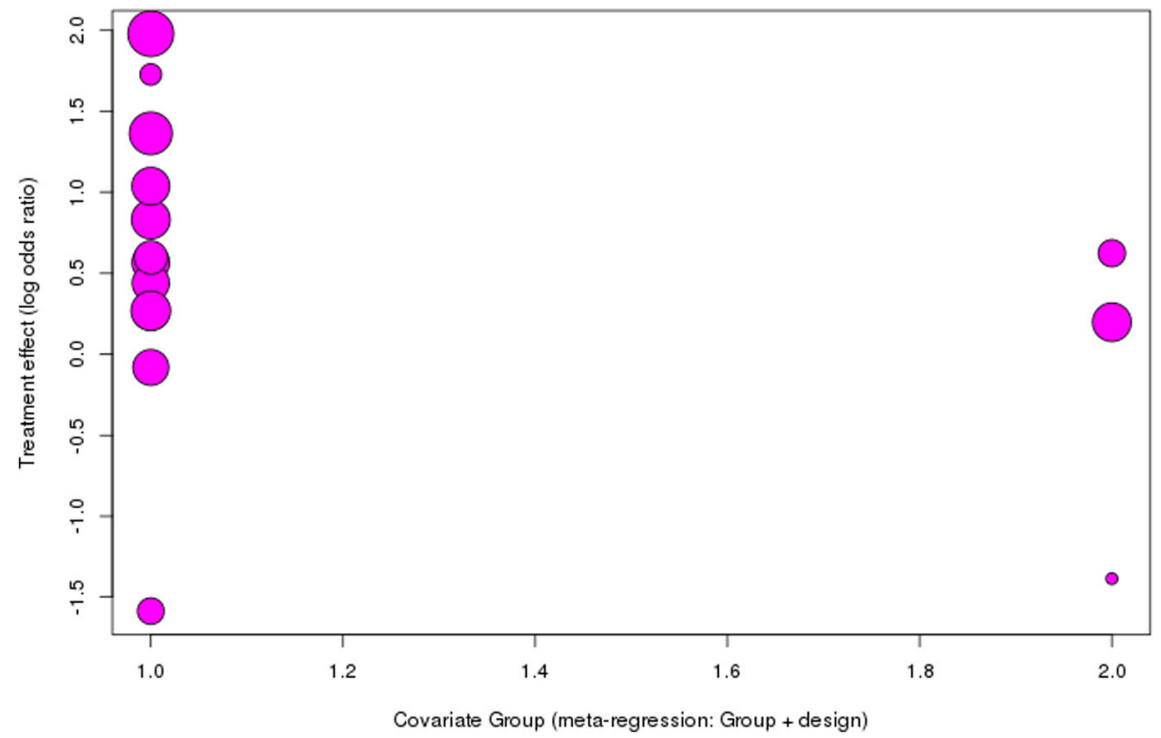

Fig. 4 a Forest plot of odds ratio with 95\% confidence intervals in meniscus tear. The gray squares represent the results of each study. Ends of the horizontal bars represent 95\% confidence intervals. Dark gray diamonds show the overall results of all studies. Random-effects models were used. b Plot of the meta-regression analysis indicated that single ACL injury and MLKI/prospective and retrospective design were not significant moderators of overall heterogeneity $(p=0.255)$

delayed, $4.5 \pm 0.52$ vs $4.4 \pm 0.66 ; p=0.28, \quad$ NMD -0.1 [95\% CI -0.54 to 0.34$]$ ).

\section{Sensitivity analysis}

We performed a meta-analysis with the results of meniscus tear and cartilage injury, after removing a single study, for the sensitivity analysis (Additional file 5: Figure S5 and Additional file 6: Figure S6). The results of the sensitivity analysis were similar to those of the initial analysis.

\section{Discussion}

The most important finding of this meta-analysis was that the delayed ligament surgery group was significantly found to have a higher risk of meniscus tear and cartilage injury and decreased Lysholm and Tegner scores 

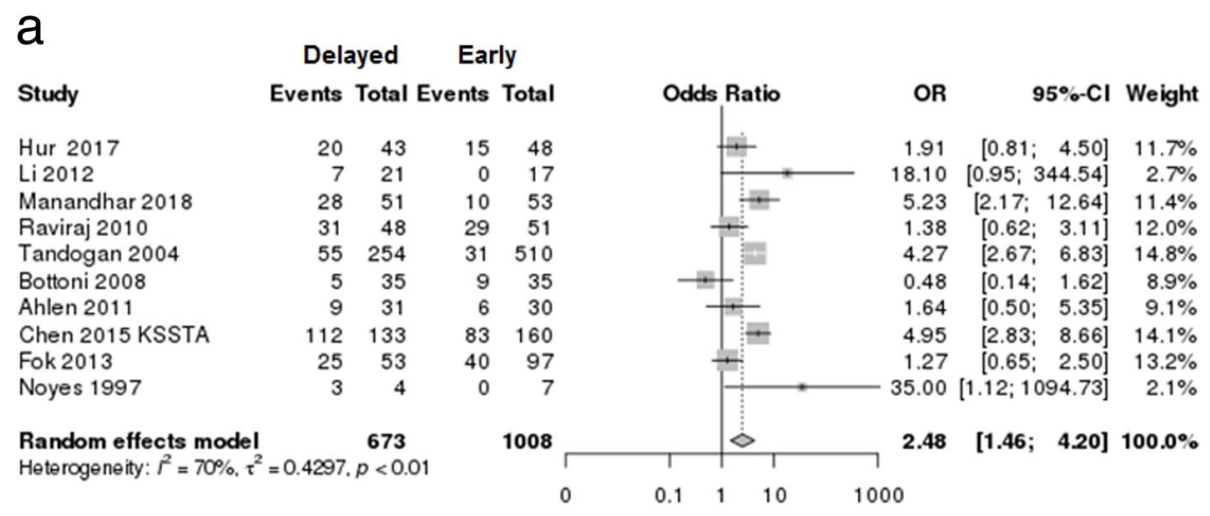

b

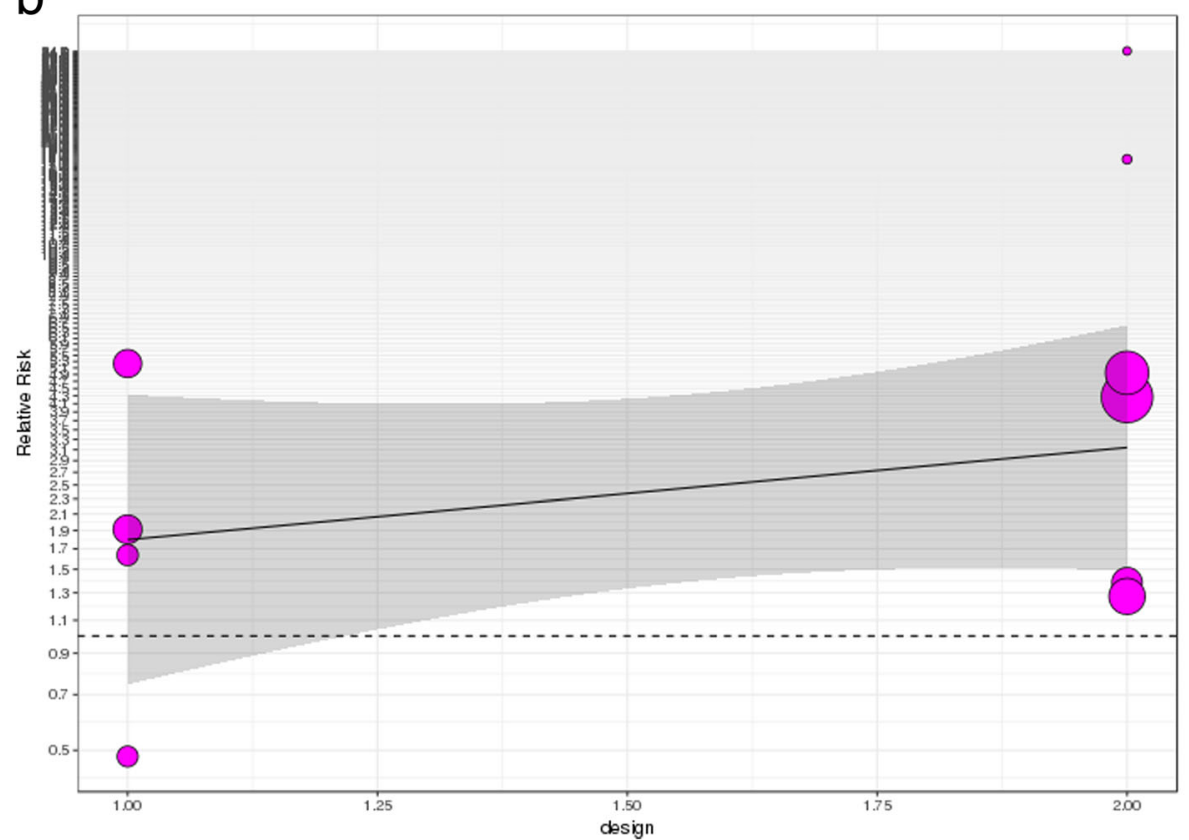

Fig. 5 a Forest plot of odds ratio with 95\% confidence intervals in cartilage injury. The gray squares represent the results of each study. Ends of the horizontal bars represent 95\% confidence intervals. Dark gray diamonds show the overall results of all studies. Random-effects models were used. $\mathbf{b}$ Plot of the meta-regression analysis indicated that prospective and retrospective design were not significant moderators of overall heterogeneity $(p=0.336)$

compared to the early ligament surgery group. In single ACL injury, a high risk of meniscus tear and decreased Lysholm and Tegner scores were found in the delayed surgery group. In MLKI, only the Lysholm score was decreased in the delayed surgery group. However, the high risk of bias due to the low level of evidence studies was also affected by the results of clinical outcomes (Lysholm and Tegner scores), and the clinical relevances of these results are still questionable.

Several previous systemic reviews and meta-analyses reported that the timing of the ACL reconstruction would not affect the outcomes [34, 44, 45], but other meta-analyses with high levels of evidence reported similar but somewhat superior results for early ACL reconstruction compared to delayed surgery [25, 60]. Recent studies, other than meta-analyses, reported that early ACL reconstruction showed better clinical results due to rapid restoration of stability and function and less risk of meniscus and cartilage injury than delayed ACL reconstruction $[24,41,42,61,62]$. In a recent meta-analysis of MLKI [4], early ligament reconstruction was recommended because of superior patient-reported and clinical outcomes. Of all patients undergoing early surgery, $31 \%$ had a normal or near-normal knee, compared to only $15 \%$ of patients undergoing delayed reconstruction [4]. However, Mook et al. [1] found worse outcomes in terms of stiffness, anterior stability, and clinical outcomes in the early surgery group.

The studies on MLKI are extremely heterogeneous, so the results might change according to the inclusion 


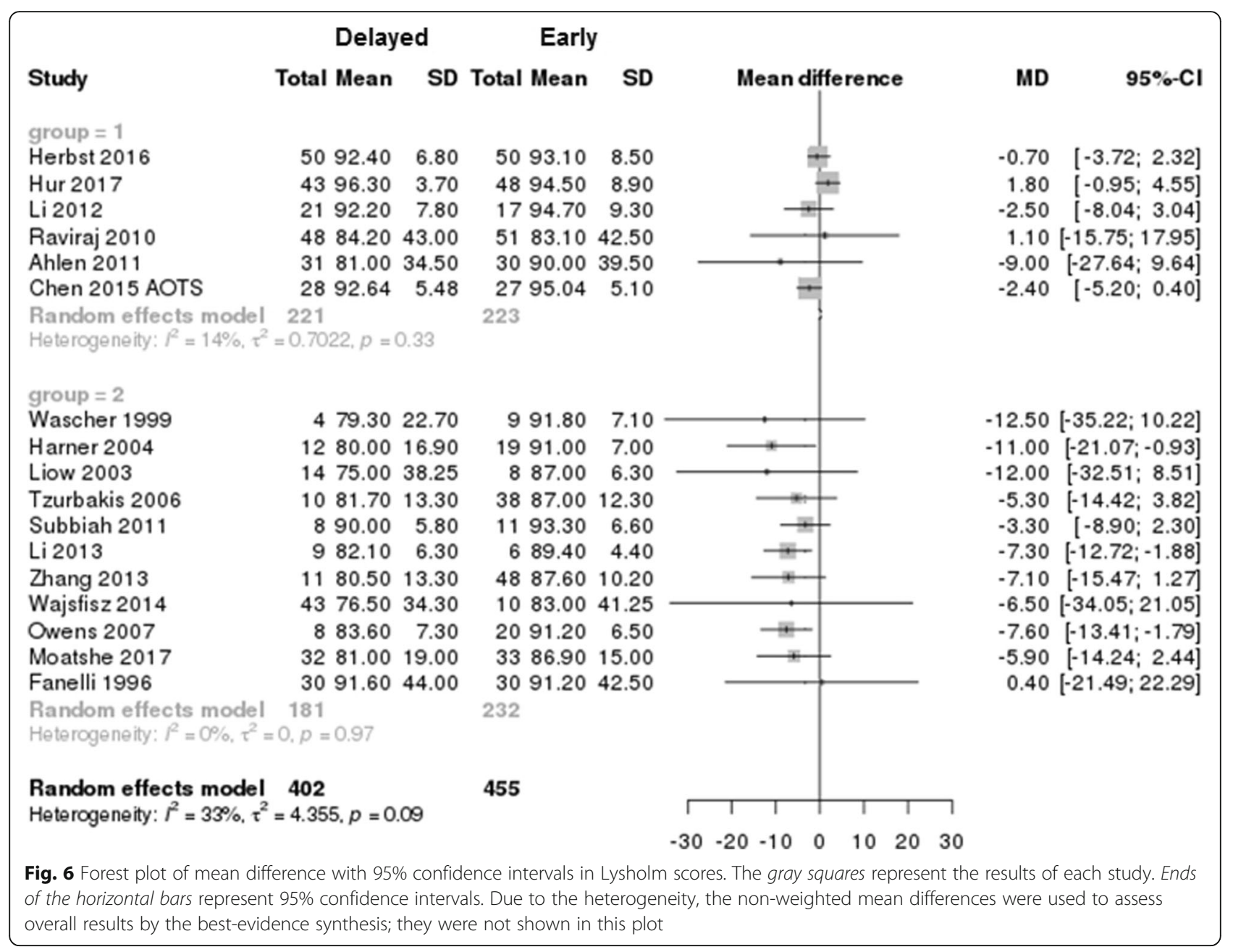

criteria. Nonetheless, all relevant articles were focused on the clinical or stability outcomes, not the incidence of meniscus tear and cartilage injury in early vs delayed surgery and the differences between single ACL injury and MLKI. Although Ferguson et al. [20] performed a meta-analysis on ACL injuries, including meniscus tear and cartilage injury, only six studies with various study designs were included. Hohmann et al. [11] performed a meta-analysis on MLKI but did not assess the outcomes of meniscus or cartilage injury and comparison with single ACL injury, which could be a control group with well-known results. Thus, it was useful to perform a meta-analysis and systemic review with studies including meniscus tear and cartilage injury results to assess the differences between early and delayed ligament reconstructions and the subgroup differences between MLKI and single ACL injury.

This systemic review including meta-analysis revealed that early ligament reconstruction could result in better Lysholm and Tegner scores and lesser risk of meniscus and cartilage injury in overall ligament injuries. The overall risk of meniscus tear and cartilage injury in the delayed surgery group was significantly increased compared to that in the early surgery group (meniscus, OR $1.73, p=0.015$; cartilage, OR 2.48, $p=0.0007$ ), and this trend was found mostly in patients with a single ACL injury (Figs. 4a and 5a). These results are similar to those in studies on ACL injury indicating that chronic ACL injuries increase the risk of meniscus and cartilage injuries, which were recognized as predictors of osteoarthritis in the long-term follow-up [24, 41, 42, 61-63].

However, there were also studies that showed no differences between early and delayed surgeries [34, 44, 45]. In the recent meta-analysis by Ferguson et al. [20], the risk of meniscus tear or cartilage injury was not significant between the early and delayed surgery groups, although the result for cartilage injury was borderline significant $(p=0.06)$. This difference might originate from the difference in the number of included studies due to the longer inclusion period of this study. Moreover, the Tegner scores in the meta-analysis by Ferguson et al. [20] were 0.39 point greater in the early surgery group than in the delayed surgery group, which was similar to the results of our analysis $(-0.25$ [95\% CI 


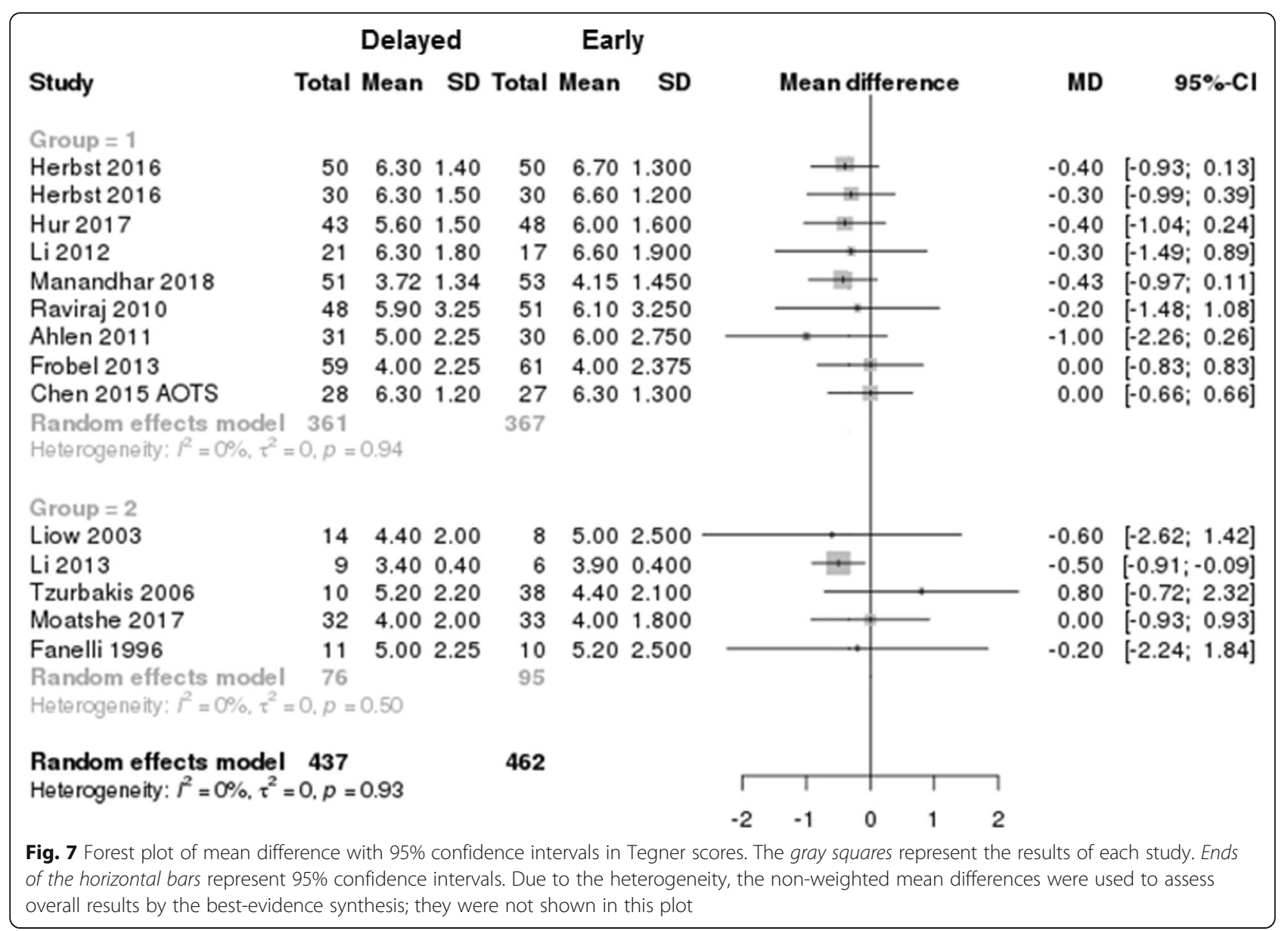

-0.45 to -0.05$])$. Although these observed scores are questionable in clinical relevance, one should address that delayed ACL surgery might have lower functional outcomes. Thus, while there were no differences in observed meniscal/chondral lesions and small differences in observed Tegner scores between the early or delayed surgery groups in previous studies [34, 45, 64], based on the results and wider included literature in this study, early intervention would be recommended to decrease the risk of developing meniscal/ cartilage lesions and potentially reduce the subsequent risk of osteoarthritis and low functional outcomes.

In MLKI, the timing of surgery did not significantly affect the incidence of meniscus tears and postoperative Tegner scores. The Lysholm scores were higher in the early surgery group than in the delayed surgery group (Fig. 6). Because all published studies on MLKI had low levels of evidence in study designs and showed heterogeneity, it is possible that future publications may change the trend of this meta-analysis by either confirming the outcome of this analysis or reversing these observed outcomes. Despite the limitations of the included study, the results of this meta-analysis are also in line with those of previous studies, which showed favorable results for early surgery [4, 30, 32, 33, 49, 65]. According to Levy [65] and Hohmann [11], higher Lysholm and IKDC scores and satisfactory final range of motion (ROM) were found in the early surgery group. McKee et al. [66] and Vicenti et al. [60] also suggested the general consensus and results of early surgery in MLKI, within the first 3 weeks, and found greater ROM in the early surgery group than in the delayed surgery group [60]. The results of our analysis, including the Lysholm score, were similar to those of previous studies $[4,65]$. Although other previous studies reported that a high incidence of arthrofibrosis was found in the early surgery group [26, 67-69], and good clinical outcomes were found in the delayed surgery group [12, $55,70,71]$, the results of this analysis and previous systemic reviews and meta-analyses $[4,60,65,66]$ suggest that early surgery of MLKI yields higher Lysholm scores with similar incidence of concomitant injuries and improved functional outcomes compared to delayed surgery.

\section{Limitations}

This study has some limitations. First, the standard of timing was different according to the studies: the 
definition of early ranged broadly up to 5 months and that of delayed ranged from 10 weeks to 24 months. Therefore, we included studies based on the author's definition of early and delayed rather than separate them as arbitrary time frames of early and delayed reconstructions by another definition. Second, all studies were included in the meta-analysis even if they did not report early vs delayed surgery as a primary outcome, especially in studies on MLKI; thus, the results were found to have substantial heterogeneity. It is extremely difficult to evaluate the clinical results on MLKI as prospective, comparative studies because of the heterogeneous nature of the injuries. Thus, we included all possible outcomes in this study and compared the results of single ACL injury as a control group. Third, relatively few studies with lower levels of evidence and small sample sizes were also major limitations, especially in studies on MLKI. Due to the searching strategy, the studies including early vs delayed and meniscus/cartilage injuries were selected for the systemic review, and the number of studies seems to be low. Moreover, in MLKIs, the different injury mechanisms and irregular knee ligament involvements and any possible combined traumas (vascular, nerve, fractures, etc.) contribute to the difficulties of analysis and obtaining consensus of treatment. The high risk of bias and heterogeneous publication of low level of evidence studies make the pooling results difficult to interpret as it is. However, in the meta-regression analysis according to the study design, there was no significant effect of the heterogeneity (Figs. 4b and 5b). Thus, we focused on the prevalence of concomitant injuries in the meta-analysis rather than the clinical outcomes, although the clinical outcomes were also reported as non-weighted means.

\section{Conclusions}

Our study suggests that delayed ACL surgery significantly resulted in higher risk of meniscus tear and cartilage injury and decreased Lysholm and Tegner scores compared to early ACL surgery. The Lysholm scores in the delayed MLKI surgery group were significantly decreased, but the risks of meniscus tear and cartilage injury in the delayed MLKI surgery group remained unclear.

\section{Supplementary Information}

The online version contains supplementary material available at https://doi. org/10.1186/s43019-020-00086-9.

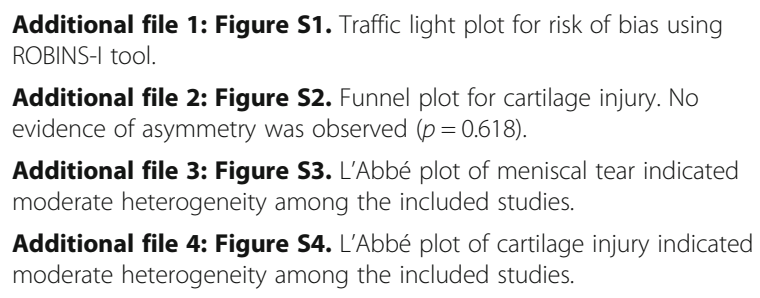

Additional file 5: Figure S5. Forest plot for sensitivity analysis of the risk of meniscus tear. The results were also significant, similar to those in the initial assessment.

Additional file 6: Figure S6. Forest plot for sensitivity analysis of the risk of cartilage injury. The results were also significant, similar to those in the initial assessment.

\section{Acknowledgements}

This research was supported by Chung-Ang University Research Grants in 2019.

\section{Authors' contributions}

SHK analyzed and interpreted the data and wrote the article. SJH analyzed the data and wrote the article. YBP, HJL, and DHK collected and interpreted the data. NP designed the article, interpreted the data, and reviewed the final article. All authors read and approved the final manuscript.

\section{Funding}

There was no funding source for this article.

\section{Availability of data and materials}

The datasets used and/or analyzed during the current study are available from the corresponding author on reasonable request.

\section{Ethics approval and consent to participate}

Not applicable.

\section{Consent for publication}

Not applicable.

\section{Competing interests}

The authors declare that they have no competing interests.

\section{Author details}

${ }^{1}$ Department of Orthopedic Surgery, Hyundae General Hospital, Chung-Ang University College of Medicine, Jinjeop-eup, Namyangju-si, Gyunggi-do, Republic of Korea. ${ }^{2}$ Department of Orthopedic Surgery, Chung-Ang University Hospital, Chung-Ang University College of Medicine, 102 Heukseok-ro, Dongjak-gu, Seoul 06973, Republic of Korea. ${ }^{3}$ Department of Orthopedic Surgery, Centre Hospitalier de Versailles, Le Chesnay, France.

Received: 7 August 2020 Accepted: 27 November 2020

Published online: 07 January 2021

\section{References}

1. Mook WR, Miller MD, Diduch DR, Hertel J, Boachie-Adjei Y, Hart JM (2009) Multiple-ligament knee injuries: a systematic review of the timing of operative intervention and postoperative rehabilitation. J Bone Joint Surg Am 91(12):2946-2957

2. Lachman JR, Rehman S, Pipitone PS (2015) Traumatic knee dislocations: evaluation, management, and surgical treatment. Orthop Clin North Am 46(4):479-493

3. Peskun CJ, Levy BA, Fanelli GC, Stannard JP, Stuart MJ, MacDonald PB et al (2010) Diagnosis and management of knee dislocations. Phys Sportsmed 38(4):101-111

4. Atkins D, Best D, Briss PA, Eccles M, Falck-Ytter Y, Flottorp S et al (2004) Grading quality of evidence and strength of recommendations. BMJ 328(7454):1490

5. Fanelli GC (2019) Multiple ligament injured knee: initial assessment and treatment. Clin Sports Med 38(2):193-198

6. Fanelli GC, Orcutt DR, Edson CJ (2005) The multiple-ligament injured knee: evaluation, treatment, and results. Arthroscopy 21(4):471-486

7. Wilson SM, Mehta N, Do HT, Ghomrawi H, Lyman S, Marx RG (2014) Epidemiology of multiligament knee reconstruction. Clin Orthop Relat Res 472(9):2603-2608

8. Liow RY, McNicholas MJ, Keating JF, Nutton RW (2003) Ligament repair and reconstruction in traumatic dislocation of the knee. J Bone Joint Surg Br 85(6):845-851 
9. Harner CD, Waltrip RL, Bennett CH, Francis KA, Cole B, Irrgang JJ (2004) Surgical management of knee dislocations. J Bone Joint Surg Am 86(2):262273

10. Tzurbakis M, Diamantopoulos A, Xenakis T, Georgoulis A (2006) Surgical treatment of multiple knee ligament injuries in 44 patients: $2-8$ years follow-up results. Knee Surg Sports Traumatol Arthrosc 14(8):739-749

11. Hohmann E, Glatt V, Tetsworth K (2017) Early or delayed reconstruction in multi-ligament knee injuries: a systematic review and meta-analysis. Knee 24(5):909-916

12. Karataglis D, Bisbinas I, Green MA, Learmonth DJ (2006) Functional outcome following reconstruction in chronic multiple ligament deficient knees. Knee Surg Sports Traumatol Arthrosc 14(9):843-847

13. Shelbourne KD, Patel DV (1995) Timing of surgery in anterior cruciate ligament-injured knees. Knee Surg Sports Traumatol Arthrosc 3(3):148-156

14. Chen J, Gu A, Jiang H, Zhang W, Yu X (2015) A comparison of acute and chronic anterior cruciate ligament reconstruction using LARS artificial ligaments: a randomized prospective study with a 5-year follow-up. Arch Orthop Trauma Surg 135(1):95-102

15. Wasilewski SA, Covall DJ, Cohen S (1993) Effect of surgical timing on recovery and associated injuries after anterior cruciate ligament reconstruction. Am J Sports Med 21(3):338-342

16. Cipolla M, Scala A, Gianni E, Puddu G (1995) Different patterns of meniscal tears in acute anterior cruciate ligament $(A C L)$ ruptures and in chronic $A C L-$ deficient knees. Classification, staging and timing of treatment. Knee Surg Sports Traumatol Arthrosc 3(3):130-134

17. Lee YS, Lee OS, Lee SH, Hui TS (2018) Effect of the timing of anterior cruciate ligament reconstruction on clinical and stability outcomes: a systematic review and meta-analysis. Arthroscopy 34(2):592-602

18. Smith TO, Davies L, Hing CB (2010) Early versus delayed surgery for anterior cruciate ligament reconstruction: a systematic review and meta-analysis. Knee Surg Sports Traumatol ArthrosC 18(3):304-311

19. Andernord D, Karlsson J, Musahl V, Bhandari M, Fu FH, Samuelsson K (2013) Timing of surgery of the anterior cruciate ligament. Arthroscopy 29(11): 1863-1871

20. Ferguson D, Palmer A, Khan S, Oduoza U, Atkinson H (2019) Early or delayed anterior cruciate ligament reconstruction: is one superior? A systematic review and meta-analysis. Eur J Orthop Surg Traumatol 29(6): 1277-1289

21. Brambilla L, Pulici L, Carimati G, Quaglia A, Prospero E, Bait C et al (2015) Prevalence of associated lesions in anterior cruciate ligament reconstruction: correlation with surgical timing and with patient age, sex, and body mass index. Am J Sports Med 43(12):2966-2973

22. Krutsch W, Zellner J, Baumann F, Pfeifer C, Nerlich M, Angele P (2017) Timing of anterior cruciate ligament reconstruction within the first year after trauma and its influence on treatment of cartilage and meniscus pathology. Knee Surg Sports Traumatol Arthrosc 25(2):418-425

23. Herbst E, Hoser C, Gföller P, Hepperger C, Abermann E, Neumayer K et a (2017) Impact of surgical timing on the outcome of anterior cruciate ligament reconstruction. Knee Surg Sports Traumatol Arthrosc 25(2):569-577

24. Granan LP, Bahr R, Lie SA, Engebretsen $L$ (2009) Timing of anterior cruciate ligament reconstructive surgery and risk of cartilage lesions and meniscal tears: a cohort study based on the Norwegian National Knee Ligament Registry. Am J Sports Med 37(5):955-961

25. Raviraj A, Anand A, Kodikal G, Chandrashekar M, Pai S (2010) A comparison of early and delayed arthroscopically-assisted reconstruction of the anterior cruciate ligament using hamstring autograft. J Bone Joint Surg Br 92(4):521-526

26. Shelbourne KD, Wilckens JH, Mollabashy A, DeCarlo M (1991) Arthrofibrosis in acute anterior cruciate ligament reconstruction. The effect of timing of reconstruction and rehabilitation. Am J Sports Med 19(4):332-336

27. Liberati A, Altman DG, Tetzlaff J, Mulrow C, Gotzsche PC, loannidis JP et al (2009) The PRISMA statement for reporting systematic reviews and metaanalyses of studies that evaluate health care interventions: explanation and elaboration. PLOS Med 6(7):e1000100

28. Booth A, Clarke M, Dooley G, Ghersi D, Moher D, Petticrew M et al (2012) The nuts and bolts of PROSPERO: an international prospective register of systematic reviews. Syst Rev 1:2

29. Spindler KP, Kuhn JE, Dunn W, Matthews CE, Harrell FE Jr, Dittus RS (2005) Reading and reviewing the orthopaedic literature: a systematic, evidencebased medicine approach. J Am Acad Orthop Surg 13(4):220-229

30. Downs SH, Black N (1998) The feasibility of creating a checklist for the assessment of the methodological quality both of randomised and non- randomised studies of health care interventions. J Epidemiol Community Health 52(6):377-384

31. McGuinness LA. robvis: an R package and web application for visualising risk-of-bias assessments. 2019 [updated 2019/07/01]. https://github.com/ mcguinlu/robvis

32. Sterne JA, Hernan MA, Reeves BC, Savovic J, Berkman ND, Viswanathan M et al (2016) ROBINS-I: a tool for assessing risk of bias in non-randomised studies of interventions. BMJ 355:14919

33. Egger M, Davey Smith G, Schneider M, Minder C (1997) Bias in meta-analysis detected by a simple, graphical test. BMJ 315(7109):629-634

34. Fragkos KC, Tsagris M, Frangos CC (2014) Publication bias in meta-analysis: confidence intervals for Rosenthal's fail-safe number. Int Sch Res Notices 2014:825383

35. Peters JL, Sutton AJ, Jones DR, Abrams KR, Rushton L (2007) Performance of the trim and fill method in the presence of publication bias and betweenstudy heterogeneity. Stat Med 26(25):4544-4562

36. Slavin RE (1995) Best evidence synthesis: an intelligent alternative to metaanalysis. J Clin Epidemiol 48(1):9-18

37. Fok AWM, Yau WP (2013) Delay in ACL reconstruction is associated with more severe and painful meniscal and chondral injuries. Knee Surg Sports Traumatol Arthrosc 21(4):928-933

38. Hur Cl, Song EK, Kim SK, Lee SH, Seon JK (2017) Early anterior cruciate ligament reconstruction can save meniscus without any complications. Indian J Orthop 51(2):168-173

39. Li B, Bai L, Fu Y, Wang G, He M, Wang J (2012) Effect of timing of surgery in partially injured ACLs. Orthopedics 35(5):408-412

40. Manandhar RR, Chandrashekhar K, Kumaraswamy V, Sahanand S, Rajan D (2018) Functional outcome of an early anterior cruciate ligament reconstruction in comparison to delayed: are we waiting in vain? J Clin Orthop Trauma 9(2):163-166

41. Nikolic DK (1998) Lateral meniscal tears and their evolution in acute injuries of the anterior cruciate ligament of the knee. Arthroscopic analysis. Knee Surg Sports Traumatol Arthrosc 6(1):26-30

42. Meighan AAS, Keating JF, Will E (2003) Outcome after reconstruction of the anterior cruciate ligament in athletic patients. J Bone Joint Surg Br 85-B(4): $521-524$

43. Ahlen M, Liden M (2011) A comparison of the clinical outcome after anterior cruciate ligament reconstruction using a hamstring tendon autograft with special emphasis on the timing of the reconstruction. Knee Surg Sports Traumatol Arthrosc 19(3):488-494

44. Frobell RB, Roos HP, Roos EM, Roemer FW, Ranstam J, Lohmander LS (2013) Treatment for acute anterior cruciate ligament tear: five year outcome of randomised trial. BMJ 346(Jan 24):f232

45. Frobell RB, Roos EM, Roos HP, Ranstam J, Lohmander LS (2010) A randomized trial of treatment for acute anterior cruciate ligament tears. $\mathrm{N}$ Engl J Med 363(4):331-342

46. Chen G, Tang X, Li Q, Zheng G, Yang T, Li J (2015) The evaluation of patient-specific factors associated with meniscal and chondral injuries accompanying ACL rupture in young adult patients. Knee Surg Sports Traumatol Arthrosc 23(3):792-798

47. Bottoni CR, Liddell TR, Trainor TJ, Freccero DM, Lindell KK (2008) Postoperative range of motion following anterior cruciate ligament reconstruction using autograft hamstrings: a prospective, randomized clinical trial of early versus delayed reconstructions. Am J Sports Med 36(4): 656-662

48. Tandogan RN, Taşer Ö, Kayaalp A, Taşkıran E, Pınar H, Alparslan B et al (2004) Analysis of meniscal and chondral lesions accompanying anterior cruciate ligament tears: relationship with age, time from injury, and level of sport. Knee Surg Sports Traumatol Arthrosc 12(4):262-270

49. Wascher DC, Becker JR, Dexter JG, Blevins FT (1999) Reconstruction of the anterior and posterior cruciate ligaments after knee dislocation. Results using fresh-frozen nonirradiated allografts. Am J Sports Med 27(2):189-196

50. Subbiah M, Pandey V, Rao SK, Rao S (2011) Staged arthroscopic reconstructive surgery for multiple ligament injuries of the knee. J Orthop Surg (Hong Kong) 19(3):297-302

51. Li X, Liu T (2013) Surgical management of multiple knee ligament injuries. Eur J Orthop Surg Traumatol 23(6):691-697

52. Zhang Y, Zhang X, Hao Y, Zhang YM, Wang M, Zhou Y (2013) Surgical management of the multiple-ligament injured knee: a case series from Chongqing, China and review of published reports. Orthop Surg 5(4):239249 
53. Wajsfisz A, Bajard X, Plaweski S, Djian P, Demey G, Limozin R et al (2014) Surgical management of combined anterior or posterior cruciate ligament and posterolateral corner tears: for what functional results? Orthop Traumatol Surg Res 100(8 Suppl):S379-S383

54. Owens BD, Neault M, Benson E, Busconi BD (2007) Primary repair of knee dislocations: results in 25 patients (28 knees) at a mean follow-up of four years. J Orthop Trauma 21(2):92-96

55. Fanelli GC, Giannotti BF, Edson CJ (1996) Arthroscopically assisted combined posterior cruciate ligament/posterior lateral complex reconstruction. Arthroscopy 12(5):521-530

56. Tardy N, Boisrenoult P, Teissier P, Steltzlen C, Beaufils P, Pujol N (2017) Clinical outcomes after multiligament injured knees: medial versus lateral reconstructions. Knee Surg Sports Traumatol Arthrosc 25(2):524-531

57. Noyes FR, Barber-Westin SD (1997) Reconstruction of the anterior and posterior cruciate ligaments after knee dislocation. Use of early protected postoperative motion to decrease arthrofibrosis. Am J Sports Med 25(6): 769-778

58. Krych AJ, Sousa PL, King AH, Engasser WM, Stuart MJ, Levy BA (2015) Meniscal tears and articular cartilage damage in the dislocated knee. Knee Surg Sports Traumatol Arthrosc 23(10):3019-3025

59. Moatshe G, Dornan GJ, Ludvigsen T, Loken S, LaPrade RF, Engebretsen L (2017) High prevalence of knee osteoarthritis at a minimum 10-year followup after knee dislocation surgery. Knee Surg Sports Traumatol Arthrosc 25(12):3914-3922

60. Vicenti G, Solarino G, Carrozzo M, De Giorgi S, Moretti L, De Crescenzo A et al (2019) Major concern in the multiligament-injured knee treatment: a systematic review. Injury 50(Suppl 2):S89-S94

61. Englund M, Lohmander LS (2004) Risk factors for symptomatic knee osteoarthritis fifteen to twenty-two years after meniscectomy. Arthritis Rheum 50(9):2811-2819

62. Lohmander LS, Englund PM, Dahl LL, Roos EM (2007) The long-term consequence of anterior cruciate ligament and meniscus injuries: osteoarthritis. Am J Sports Med 35(10):1756-1769

63. Drogset JO, Grontvedt T (2002) Anterior cruciate ligament reconstruction with and without a ligament augmentation device: results at 8-year followup. Am J Sports Med 30(6):851-856

64. Church S, Keating JF (2005) Reconstruction of the anterior cruciate ligament: timing of surgery and the incidence of meniscal tears and degenerative change. J Bone Joint Surg Br 87(12):1639-1642

65. Levy BA, Dajani KA, Whelan DB, Stannard JP, Fanelli GC, Stuart MJ et al (2009) Decision making in the multiligament-injured knee: an evidencebased systematic review. Arthroscopy 25(4):430-438

66. MCKee L, Ibrahim MS, Lawrence T, Pengas IP, Khan WS (2014) Current concepts in acute knee dislocation: the missed diagnosis? Open Orthop I 8: 162-167

67. Harner CD, Irrgang JJ, Paul J, Dearwater S, Fu FH (1992) Loss of motion after anterior cruciate ligament reconstruction. Am J Sports Med 20(5):499-506

68. Mohtadi NG, Webster-Bogaert S, Fowler PJ (1991) Limitation of motion following anterior cruciate ligament reconstruction. A case-control study. Am J Sports Med 19(6):620-624 discussion 4-5

69. Shelbourne KD, Nitz P (1990) Accelerated rehabilitation after anterior cruciate ligament reconstruction. Am J Sports Med 18(3):292-299

70. Fanelli GC, Edson CJ (2002) Arthroscopically assisted combined anterior and posterior cruciate ligament reconstruction in the multiple ligament injured knee: 2- to 10-year follow-up. Arthroscopy 18(7):703-714

71. Fanelli GC, Edson CJ (2004) Combined posterior cruciate ligamentposterolateral reconstructions with Achilles tendon allograft and biceps femoris tendon tenodesis: 2- to 10-year follow-up. Arthroscopy 20(4):339345

\section{Publisher's Note}

Springer Nature remains neutral with regard to jurisdictional claims in published maps and institutional affiliations.

Ready to submit your research? Choose BMC and benefit from:

- fast, convenient online submission

- thorough peer review by experienced researchers in your field

- rapid publication on acceptance

- support for research data, including large and complex data types

- gold Open Access which fosters wider collaboration and increased citations

- maximum visibility for your research: over $100 \mathrm{M}$ website views per year

At BMC, research is always in progress.

Learn more biomedcentral.com/submissions 